\title{
IBERIRHYNCHIIDAE NUEVA FAMILIA DE RINCONÉLIDOS ANCISTRORHYNCHOIDEOS (BRAQUIÓPODOS) DEL ORDOVÍCICO MEDIO AL DEVÓNICO INFERIOR DE EUROAMÉRICA Y GONDWANA
}

\author{
Jenaro L. GARCÍA-ALCALDE \\ Departamento de Geología (Área de Paleontología) de la Universidad de Ovie- \\ do, c/ Jesús Arias de Velasco, s/n., 33005 Oviedo. \\ email: jalcalde@geol.uniovi.es
}

García-Alcalde, J. L. 2009. Iberirhynchiidae nueva familia de rinconélidos ancistrorhynchoideos (braquiópodos) del Ordovícico Medio al Devónico Inferior de Euroamérica y Gondwana. [Iberirhynchiidae, a new family of ancistrorhynchoid rhynchonellids (Brachiopoda) from the Middle Ordovician to the Lower Devonian of Euroamerica and Gondwana.] Revista Española de Paleontología, 24 (2), 149-169. ISSN 0213-6937

\section{EXTENDED ABSTRACT}

The type species of the genus Iberirhynchia Drot \& Westbroek, 1966, I. santaluciensis Drot \& Westbroek, 1966 is a small, subtriangular, dorsibiconvex, completely costate rhynchonellid, with ventral vertical dental plates and a dorsal cup, supported on a median partition, covered anteriorly by a connectivum.

After their morphological characters Iberirhynchia has usually been included in the Family Trigonirhynchiidae Schmidt, 1965 (Superfamily Rhynchotrematoidea Schuchert, 1913). However the Iberirhynchia median dorsal partition structure departs clearly from that of Trigonirhynchia Cooper, 1942 and related genera.

These last forms have a true median septum provided by an axial median granular calcite sheet (mediotest) which during further ontogeny became moderately thicker by apposition of secondary shell lamellae on both sides of the septum. On the contrary, the Iberirhynchia median dorsal partition is in fact a rather thick swelling of the bottom shell originated by differential secretion of secondary shell from the floor of the valve upwards (pseudoseptum).

The throughlike internal dorsal structure formed by the merging of both the crural plates and pseudoseptum was named by Rozman (1969) (fide Havlíček \& Štorch, 1990) a pseudoseptalium and its occurrence was used to characterize the Subfamily Rostricellulinae against other trigonirhynchiid genera provided by a conventional median septum and septalium. The pseudoseptum becomes also evident in the shell exterior by the occurrence of a marked dorsal median umbonal depression that extends distally sometimes as far as to reach the frontal commissure. There is a great anatomical difference between the drastic folding of the dorsal outer epithelium along the plane of symmetry that gives rise to the trigonirhynchiid median septum and the simple apposition of secondary shell lamellae that originates a pseudoseptum.

Havlíček \& Štorch (1990) and García-Alcalde (1998) considered long ago the occurrence of a pseudoseptalium as important as to reassigning several trigonirhynchiid taxa to the Family Oligorhynchiidae Cooper, 1956, belonging to another different Superfamily unit, the Ancistrorhynchoidea Cooper, 1956. Recent systematic studies by Savage have limited the concept of Oligorhynchiidae to very small, paucicostate, elongated rinchonellids, lacking of septalial or pseudoseptalial dorsal structures although with a tendency to develop a pseudoseptum in the floor of the dorsal valve. In these conditions, all the forms with pseudoseptate structures are considered provisionally as belonging to the Superfamily Ancistrorhynchoidea, although without ruling out that the pseudoseptate forms with a complete, usually covered pseudoseptalium lacking of a cardinal process could be grouped in a new superfamily unit.

Iberirhynchia is proposed herein as the type of the new Family Iberirhynchiidae that combines features usual in Trigonirhynchiidae Schmidt, 1965 with the occurrence of a well developed, usually covered pseudoseptalium. To that family and to the new Subfamily Iberirhynchiinae would belong, besides the type genus, at least Tectogonotoechia García-Alcalde, 1998, Ancillotoechia Havlíček, 1959, Aratoechia Havlíček, 1982 and Microsphaeridiorhynchus Sartenaer, 1970.

These genera are characterized by the occurrence of a complet, covered pseudoseptalium, vertical dental plates and simple radial ornamentation. Myrmirhynx Havlíček, 1982 and Sufetirhynchia Havlíček 1982, integrate another new Subfamily Myrmirhynxinae, close to Iberirhynchiinae but with divided radial ornamentation. The Iberirhynchiidae would be completed by the subfamilies Rostricellulinae Rozman, 1969, lacking of connectivum, and Virginiatinae Amsden, 1974, with a partial cover plate, that lacks sometimes. 
According both the ages and the paleogeographical distribution of the iberhynchiid genera, the Family Iberirhynchiidae started at the middle Ordovician with a basal trunk constituted by the cosmopolitan genus Rostricellula Ulrich \& Cooper, 1942 and the other upper Ordovician-lower Silurian Rostricellulinae. Virginiatinae Amsden, 1974 could in turn derivate from the Rostricellulinae along the upper Ordovician. Later on, along the Silurian appeared both the Iberirhynchiinae nov subfam. (Wenlock-upper Emsian), and the Myrmirhynxinae nov. subfam. (Wenlock-Lochkovian). Ancillotoechia, a Silurian to lowermost Devonian, cosmopolitan genus, was probably the ancestral, more generalized Iberirhynchiinae form. In the other hand, Myrmirhynxinae formed a marginal, specialized branch, of uncertain origin, only known in Bohemia. The Silurian Family Niorhynicidae Savage, 1996, with complet, covered or not, pseudoseptalium, but lacking of dental plates could be either a sister group of Iberihynchiidae among the Ancistrorhynchoidea or form with them a new superfamily unit. Iberirhynchia (upper Emsian) is the younger iberhynchiid genus, but its origin and relationships with other Iberirhynchiinae are not well known at the present.

Iberirhynchia santaluciensis is redescribed from: a) new, numerous possible topotypic specimens, coming from the Pico Aguasalio region, León province (N Spain); b) several specimens from different localities in Asturias and León provinces; and c) a small original sample that we have got on loan from the Rijksmuseum van Geologie en Mineralogie of Leiden, Nederland.

In this redescription several problems concerning the type material, locus typicus and stratum typicum are dealt with and solved as far as possible. Ontogenetic and paleoecological observations are also given.

Shells show an allometric growth with a proportionally greater increase of width and thickness against length with the age. Neanic shells are thin, small, ventribiconvex, longer than wide or as long as wide, with sharp commissures, faintly inclined beak, acute apical angles $\left(40-60^{\circ}\right)$ and inconspicuous ventral sinus and dorsal fold; the transition to the ephebic stage is marked usually by a strong growth lamella that indicates a more or less prolonged growth stopping. The ephebic shells are dorsibiconvex, gradually thicker and clearly wider than long, with suberect beaks and apical angles greater than $60^{\circ}$, well developed ventral sinus and dorsal fold and verticalized lateral and anterior margins. Gerontic stage begins also after a marked growth stopping; gerontic shells are very thick and transverse, with high and vertical lateral sides that meet at the commissure in angles close to $180^{\circ}$, and apical angles between $70-90^{\circ}$ or more. In general, epizoans and repair damage signals are not abundant indicating a not too rough environment and a rather stable sea bottom.

Until to now, I. santaluciensis is the sole known Iberirhynchia species. Bohemian forms named Iberirhynchia nargis Havlíček \& Kukal, 1990, and Iberirhynchia sp. Havlíček 1992, are true trigonirhynchiids, with conventional, not covered septalium.

Keywords: Iberirhynchiidae nov. fam., Iberirhynchiinae nov. subfam., Myrmirhynxinae nov. subfam., Rhynchonelllida Ancistrorhynchoidea, Gondwana, Euroamerica, Middle Ordovician to Lower Devonian, Ontogeny, Paleoecology.

\section{RESUMEN}

Se propone la nueva Familia Iberirhynchiidae (Ancistrorhynchoidea), para rinconélidos anteriormente incluidos en Trigonirhynchiidae (Rhynchotrematoidea), pero con seudoseptalio, cubierto o no por un conectivo, formado por la unión de las placas crurales con un tabique medio dorsal constituido por láminas superpuestas de concha secundaria, en lugar del típico septo medio trigonirínquido, con finas láminas de calcita granular (mediotest) en la región axial, sobre las que van apareciendo hacia delante láminas de concha secundaria.

Iberirhynchiidae nov. fam. integra varias subfamilias: Iberirhynchiinae nov. subfam., con el género tipo Iberirhynchia, y los géneros Ancillotoechia, Aratoechia, Tectogonotoechia y Microsphaeridiorhynchus, con seudoseptalio completo, cubierto por un conectivo, y ornamentación radial simple, del Wenlock al Emsiense superior; Myrmirhynxinae nov. subfam., que comprende los géneros del Wenlock-Ludlow de Bohemia, Myrmirhynx y Sufetirhynchia, parecida a la anterior, pero con conchas con ornamentación dividida; Rostricellulinae, que integra géneros del Ordovícico Medio al Silúrico más bajo, carentes de conectivo y con ornamentación frecuentemente dividida, cortada por fuertes lamelas de crecimiento; y Virginiatinae, con géneros del Ordovícico Superior al Ludlow, con conectivo parcial o sin él, y ornamentación principalmente simple.

Se redescribe la especie-tipo de Iberirhynchia, I. santaluciensis, a partir de una numerosa muestra de ejemplares posiblemente topotípicos, varios especimenes de diferentes localidades asturianas y leonesas, y de otra pequeña muestra de material original prestado por el Rijksmuseum van Geologie en Mineralogie de Leiden, Holanda. En esta redescripción se abordan y se resuelven, hasta donde resulta posible, diferentes problemas relacionados con la naturaleza, procedencia y actual lugar de depósito del holotipo y demás tipos de la especie. Se aportan también diferentes observaciones paleoecológicas que muestran el desarrollo alométrico de la concha que, con 
la edad, crece más en grosor que en longitud. La relativa escasez de epizoos y de señales de reparación de las conchas, indica un medio marino relativamente tranquilo y estable. Hasta ahora, la única especie del género es la tipo, I. santaluciensis. Las formas denominadas Iberirhynchia nargis e Iberirhynchia sp. son verdaderos trigonirínquidos, con septalio convencional, no cubierto.

Palabras clave: Iberirhynchiidae nov. fam., Iberirhynchiinae nov. subfam., Myrmirhynxinae nov. subfam., Rhynchonelllida Ancistrorhynchoidea, Gondwana, Euroamérica, Ordovícico Medio a Devónico Inferior, Ontogenia, Paleoecología.

\section{INTRODUCCIÓN}

J. Drot y P. Westbroek propusieron en 1966 el nuevo género Iberirhynchia, constituido exclusivamente por su especie-tipo, Iberirhynchia santaluciensis Drot \& Westbroek, 1966, un pequeño braquiópodo del Orden Rhynchonellida, abundante y característico en niveles próximos a la transición Devónico Inferior-Devónico Medio de la Cordillera Cantábrica. Las referencias posteriores al género en Bohemia (Havlíček \& Kukal, 1990; Havlíček, 1992) corresponden a otro u otros taxones diferentes.

En origen, el género se incluyó en la Familia Trigonirhynchiidae Schmidt, 1965, donde lo mantuvieron la mayoría de autores posteriores. Savage (2002), en concreto, fijó su posición, en el Treatise on Invertebrate Paleontology, dentro de la referida familia, en la Subfamilia Trigonirhynchiinae Schmidt, 1965. Iberirhynchia posee, en efecto, características que lo acercan a los Trigonirhynchiidae: en el exterior (Figs. 1,2), concha pequeña, triangular, completamente costada, con pliegue dorsal y seno ventral desarrollados y comisura dentada, y en el interior, placas dentales verticales y tabique medio dorsal, con estructura septalial cubierta, sin apófisis cardinal (Fig. 3). Sin embargo, la estructura interna del tabique medio dorsal es radicalmente diferente a la de Trigonirhynchia Cooper, 1942 y géneros relacionados. En éstos se trata de un auténtico septo, cuya parte axial es una capa delgada de calcita granular (mediotest), de génesis y estructura muy similares a los de la concha primaria, de la que se proyectan anterolateralmente fibras de concha secundaria, flanqueando el septo en toda su longitud (Westbroek, 1967). En cambio, en Iberirhynchia dicho tabique tiene su origen en un abombamiento medio del fondo de la valva, que va ganando altura y grosor en el curso de la ontogenia por aposición de sucesivas láminas concéntricas de concha secundaria fibrosa (Figs. 3-7) (comparar, en particular, Figs. 5.a-c y 5.d-f). Este tipo de desarrollo se evidencia en el género y en muchos otros con la misma estructura, por la presencia de una marcada depresión longitudinal media, más o menos larga, en el exterior de la valva dorsal (Figs. 1-7), ausente en los trigonirínquidos convencionales.

A la copa dorsal formada por la unión de las placas crurales al tabique medio dorsal, constituido sólo por concha secundaria, la denominó Rozman (1969) "seudoseptalio", y dicha estructura le permitió separar su Subfamilia Ros- tricellulinae de otros taxones de Trigonirhynchiidae con verdadero septo medio.

Havlíček \& Štorch (1990) concedieron mucha importancia sistemática a la presencia o ausencia de seudoseptalio, hasta el punto de reasignar numerosos géneros de Trigonirhynchiidae a otra familia, la de los Oligorhynchiidae Cooper, 1956, muy alejada de la anterior y considerada hoy día, incluso, como propia de una superfamilia distinta: Ancistrorhynchoidea Cooper, 1956. En la misma línea, García-Alcalde (1998), incluyó en Oligorhynchiidae, entre otros, a Iberirhynchia y Tectogonotoechia García-Alcalde, 1998.

La reorganización sistemática propuesta por Savage $(1996,2002)$ ha limitado el concepto de Oligorhynchiidae a rinconélidos muy pequeños, paucicostados y alargados, carentes de cualquier estructura septalial o seudoseptalial, aunque con tendencia a la formación de un abombamiento mediodorsal de concha secundaria fibrosa (seudosepto). Por ello, la drástica enmienda propuesta por Havlíček \& Štorch (1990) para aquella familia, que seguimos en su día (García-Alcalde, 1998), no nos parece ahora adecuada para reflejar la realidad sistemática de las formas discutidas.

Savage $(1996,2002)$, sin embargo, ha obviado las observaciones de Rozman (1969) y Havlíček \& Štorch (1990), distribuyendo entre las diferentes subfamilias de Trigonirhynchiidae las formas con seudoseptalio. Existe, sin embargo, una diferencia anatómica profunda, señalada en parte por Westbroek (1967), entre el replegamiento profundo del epitelio externo a lo largo del plano de simetría en la valva dorsal, que da lugar al septo trigonirínquido convencional, y la simple secreción diferencial, en dicha parte de la valva, de mayor cantidad de concha secundaria, que conduciría a la formación de un seudosepto. Esta diferencia sería comparable a la que presentarían con las anteriores otras formas sin ningún tipo de tabique medio, característica a la que el propio Savage $(1996,2002)$, entre otros autores, concede importancia sistemática frecuentemente supragenérica, incluso suprafamiliar.

\section{ESQUEMA CLASIFICATORIO}

Por las razones antes indicadas, provisionalmente asignamos todas las formas con seudoseptalio o seudosepto a la Superfamilia Ancistrorhynchoidea Cooper, 1956, sin 


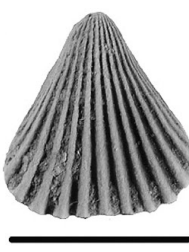

a1

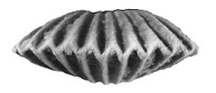

a3

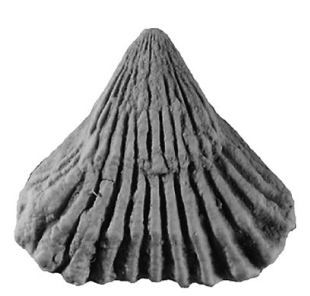

d1

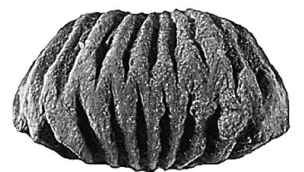

d2

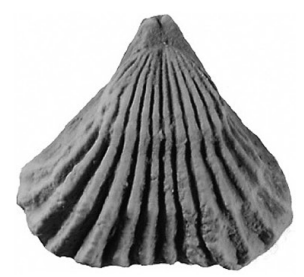

d3

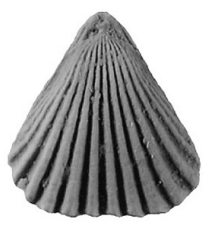

a2

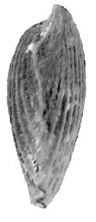

a4

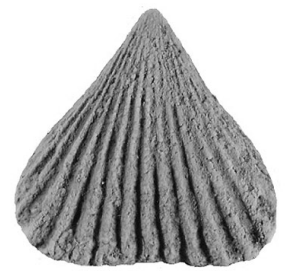

b1

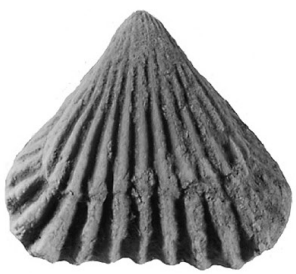

c1

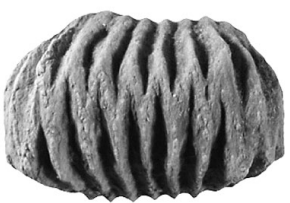

c4

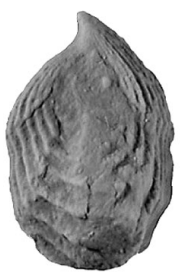

d4

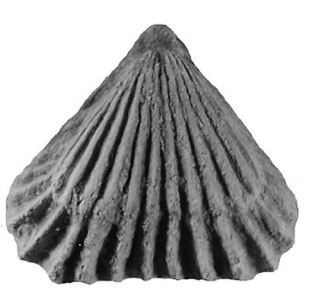

c2

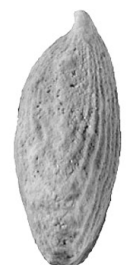

b2

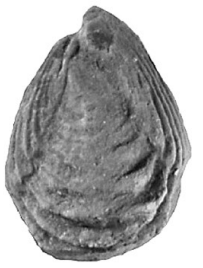

c3

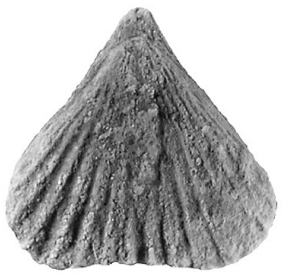

b3

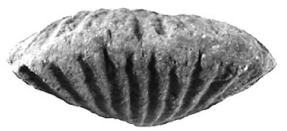

b4

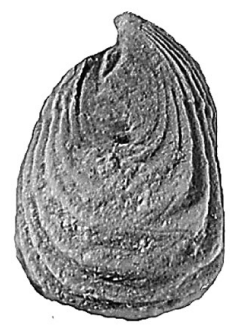

e2 e1

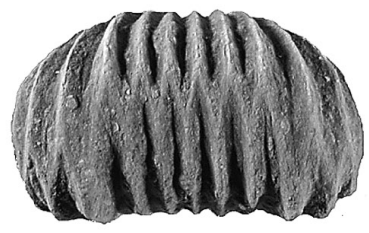

e3

1
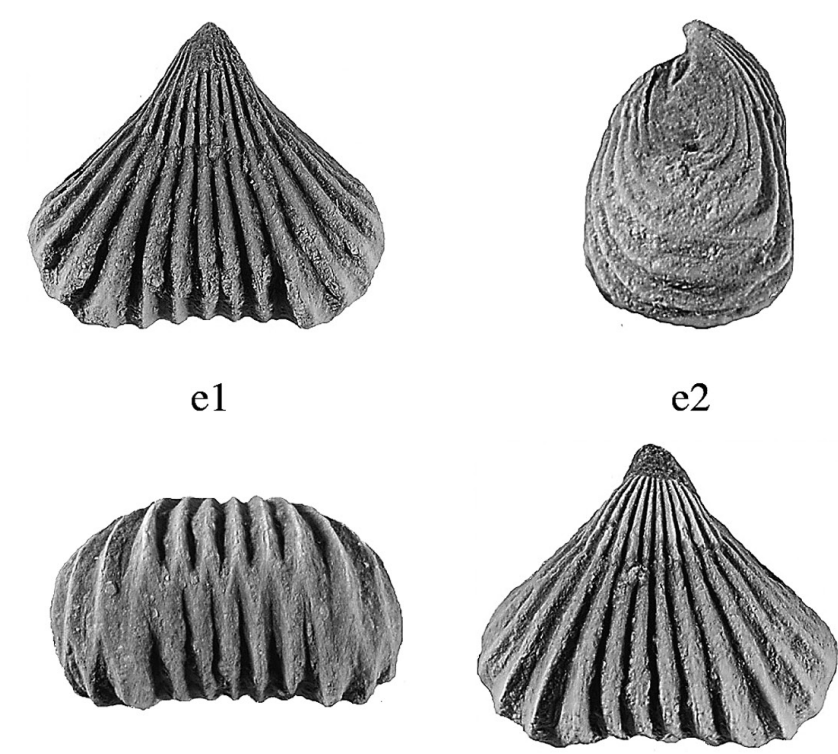

e4

Figura 1. (Barra horizontal: $5 \mathrm{~mm}$. Todos los ejemplares blanqueados con óxido de magnesio). Iberirhynchia santaluciensis Drot \& Westbroek, 1966. Arroyo de La Trapa, 500 m ESE Argovejo (Crémenes, Cistierna, provincia de León, N de España), nivel AR-SL-5, Miembro III, Fm. Santa Lucía, Intervalo Faunístico 16. a1-a4: DPO 38361-16, ejemplar juvenil, vistas ventral, dorsal, anterior y lateral. Obsérvense las comisuras cortantes, la anterior prácticamente rectimarginada y perfil ventribiconvexo. b1-b4: DPO 38361-42, ejemplar juvenil, vistas ventral, lateral, dorsal y anterior. c1-c4: DPO 38361-59, ejemplar efébico temprano, vistas ventral, dorsal, lateral y anterior. Obsérvese la detención del crecimiento en el límite entre los estadios neánico y efébico, marcado por una línea de crecimiento más fuerte que las otras. d1-d4: DPO 3836166, ejemplar efébico tardío, vistas ventral, anterior, dorsal y lateral. Obsérvense la marcada depresión media dorsal que alcanza la comisura frontal y las dos fuertes líneas de crecimiento que indican el comienzo de las fases efébica temprana y efébica tardía. La figura d1 muestra, además, alteraciones en la dirección de crecimiento de las costillas en el límite entre las fases neánica y efébica. e1-e4: DPO 38361-138, vistas ventral, lateral, anterior y dorsal.

(Horizontal bar: $5 \mathrm{~mm}$. All specimens were whitened with magnesium oxide). Iberirhynchia santaluciensis Drot \& Westbroek, 1966. La Trapa Creek, 500 m ESE Argovejo (Crémenes, Cistierna, province of León, N Spain), bed AR-SL-5, Member III, Santa Lucía Formation, Faunal Interval 16. 1-4: DPO a1-a4: young specimen, ventral, dorsal, anterior, and lateral views. See the ventribiconvex profile and the sharp commissures, the anterior one nearly rectimarginate. b1-b4: DPO 38361-42, young specimen, ventral, lateral, dorsal, and anterior views. c1-c4: DPO 38361-59, early ephebic specimen, ventral, dorsal, lateral, and anterior views. See the growth stopping between the neanic-ephebic stage boundary, marked by a strong growth line. d1-d4: DPO 38361-66, late ephebic specimen, ventral, anterior, dorsal, and lateral views. See both the marked dorsal umbonal depressio prolonged until the frontal commissure, and two strong growth lines indicating the beginning of the early ephebic, and the late ephebic stages. Figure d1 shows moreover disturbed growing vectors of the ribs at the neanic/ephebic stage boundary. e1-e4: DPO 38361-138, ventral, lateral, anterior, and dorsal views. 


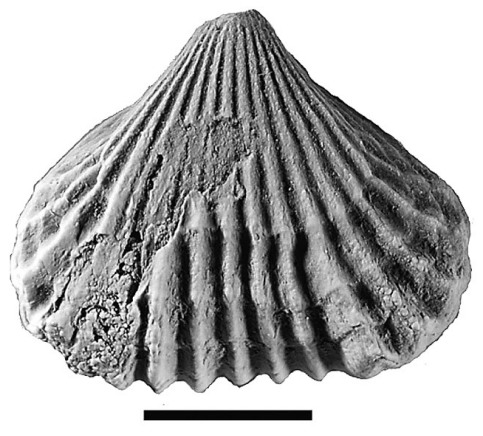

a1

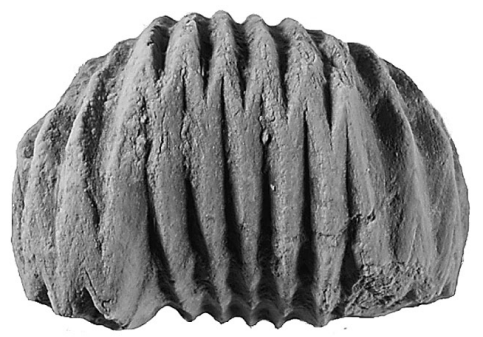

a4

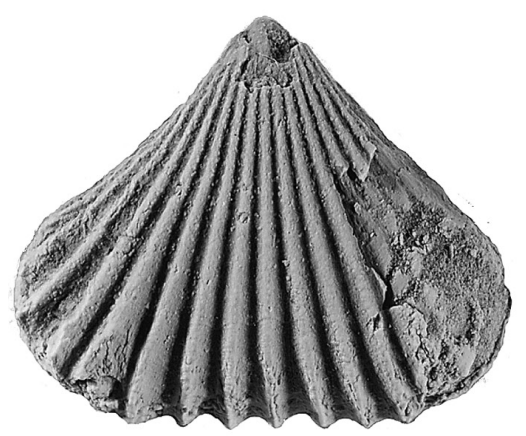

b1

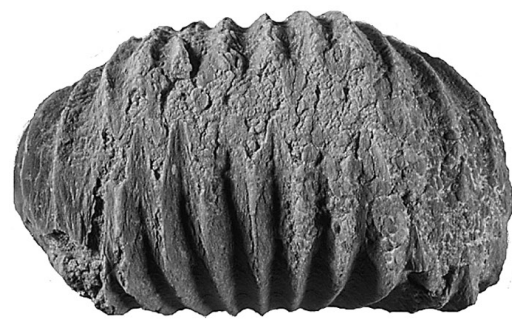

b3

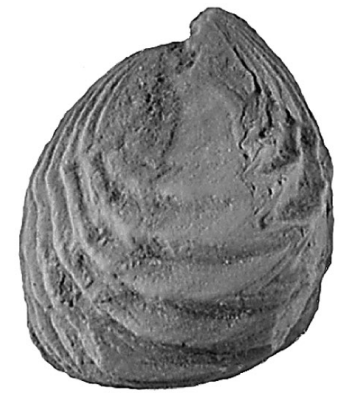

a2

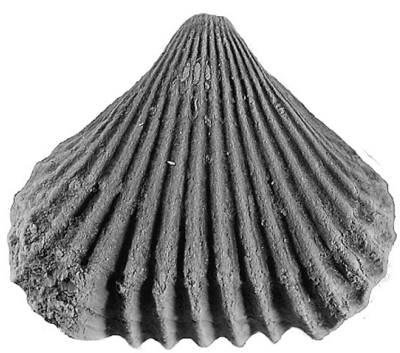

c1

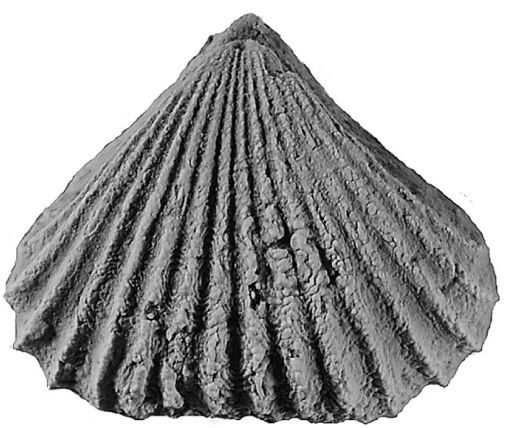

b2

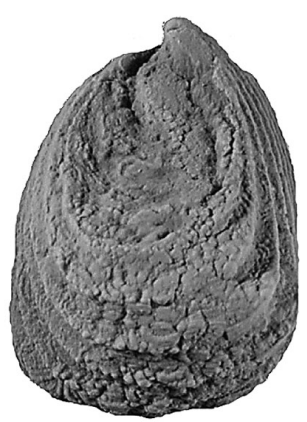

b4

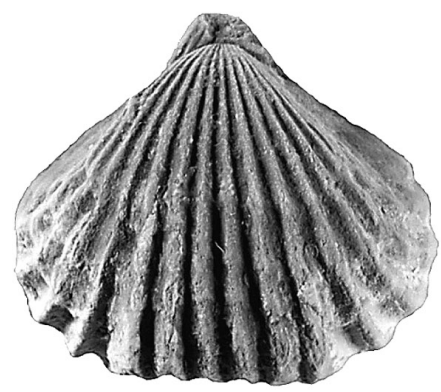

a3

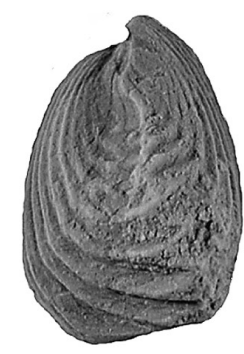

c2

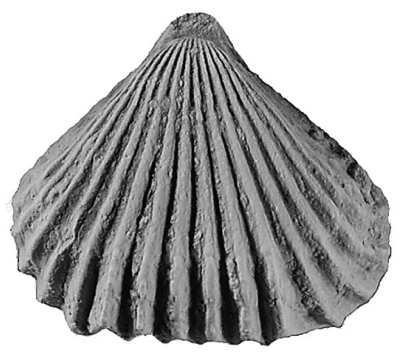

c3

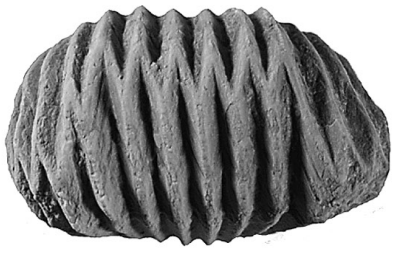

c4

Figura 2. (Barra horizontal: $5 \mathrm{~mm}$. Todos los ejemplares blanqueados con óxido de magnesio). Iberirhynchia santaluciensis Drot \& Westbroek. Ejemplares gerónticos de la misma procedencia que los de la figura 1. a1-a4: DPO 38361-207, vistas ventral, lateral, dorsal y anterior. b1-b4: DPO 38361-210, vistas ventral, dorsal, anterior y lateral. c1-c4: DPO 38361-167, vistas ventral, lateral, dorsal y anterior.

(Horizontal bar: $5 \mathrm{~mm}$. All specimens were whitened with magnesium oxide). Iberirhynchia santaluciensis Drot \& Westbroek, 1966. Gerontic specimens, same locality than figure 1. a1-a4: DPO 38361-207, ventral, lateral, dorsal, and anterior views. b1-b4: DPO 38361-210, ventral, dorsal, anterior, and lateral views. c1-c4: DPO 38361-167, ventral, lateral, dorsal, and anterior views. 

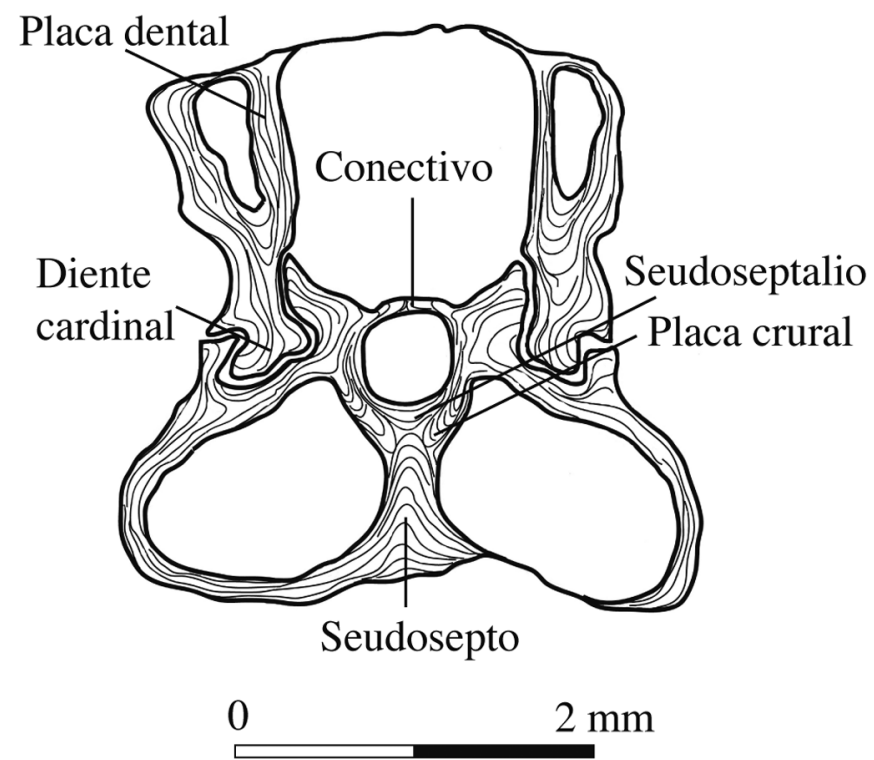

Figura 3. Iberirhynchia santaluciensis Drot \& Westbroek, 1966. Sección normal a los planos de simetría y de comisura, a 0,5 mm del ápice de la concha, mostrando la estructura del seudoseptalio y de otros órganos internos. Iberirhynchia santaluciensis Drot \& Westbroek, 1966. Section normal to the planes of symmetry and commissure, at $0.5 \mathrm{~mm}$ from the shell apex, showing the structure of dorsal pseudoseptalium and other internal organs.

descartar que las que tienen el seudoseptalio completo, usualmente cubierto por conectivo y sin apófisis cardinal, puedan en el futuro agruparse en una unidad superfamiliar nueva.

Iberirhynchia Drot \& Westbroek, 1966, es el tipo de la nueva Familia Iberirhynchiidae, que combina caracteres trigonirínquidos con la presencia de seudoseptalio bien desarrollado. A dicha familia y a la nueva Subfamilia Iberirhynchiinae pertenecerían, además del género tipo, al menos, Tectogonotoechia García-Alcalde, 1998, Ancillotoechia Havlíček, 1959, Aratoechia Havlíček, 1982, y Microsphaeridiorhynchus Sartenaer, 1970 (Fig. 7), con conectivo, placas dentales verticales y ornamentación radial esencialmente simple. Otros géneros, como Myrmirhynx Havlíček, 1982 y Sufetirhynchia Havlíček, 1982, integran una nueva unidad de los Iberirhynchiidae, los Myrmirhynxinae nov. subfam., caracterizada por la presencia constante de ornamentación radial dividida. Los Rostricellulinae Rozman, 1969, y los Virginiatinae Amsden, 1974 pertenecerían también a la nueva familia; los primeros se caracterizarían principalmente por la ausencia de conectivo, costillas a veces divididas y/o intercalares, cortadas por fuertes lamelas concéntricas de crecimiento; y los segundos, por carecer de conectivo o tenerlo incompleto (Fig. 8).

Es posible que más géneros incluidos entre los Trigonirhynchiidae, una familia excesivamente comprensiva (ver
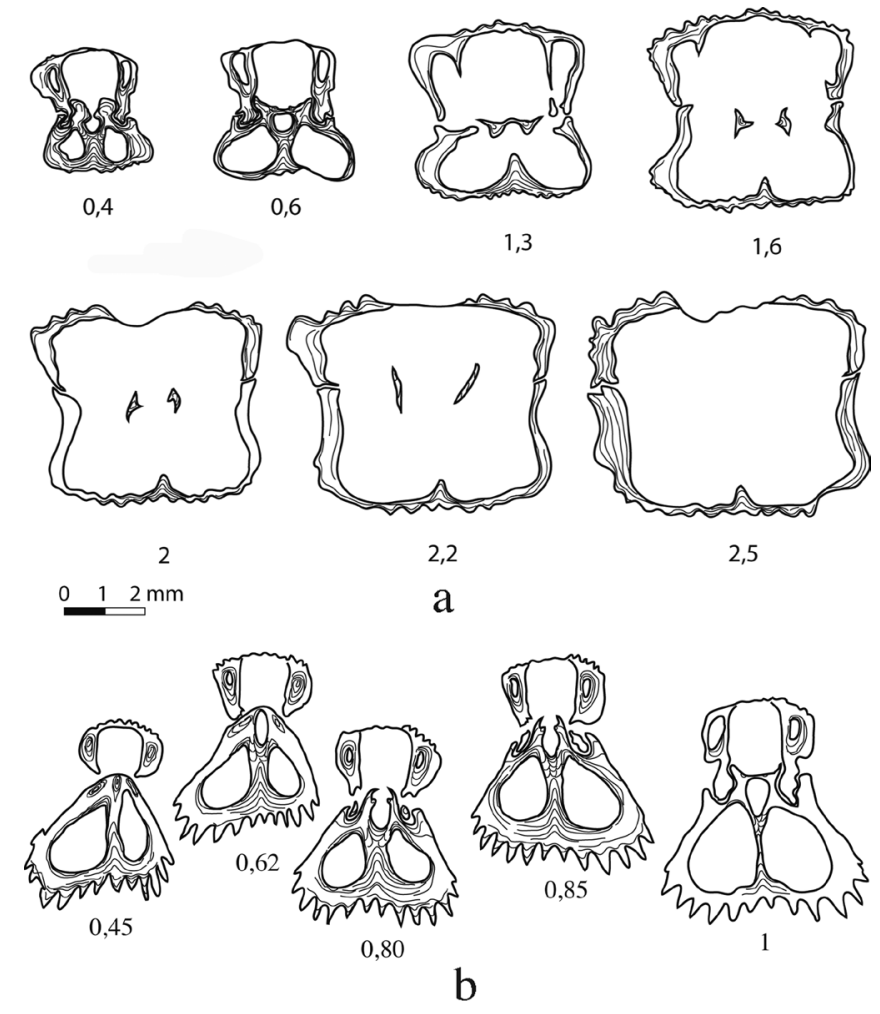

Figura 4. Dibujos con cámara clara de las secciones seriadas de Iberirhynchia santaluciensis Drot \& Westbroek, 1966. a: DPO 37188 (ejemplar de alrededor de $6 \mathrm{~mm}$ de longitud); b: DPO 38362-3 (ejemplar de $10 \mathrm{~mm}$ de longitud). Distancias medidas al ápice ventral. Camera lucida drawings of serial sections of Iberirhynchia santaluciensis Drot \& Westbroek, 1966. a: DPO 37188 (specimen ca. $6 \mathrm{~mm}$. length); $\boldsymbol{b}:$ DPO 38362-3 (specimen of $10 \mathrm{~mm}$ length). Distances measured in $\mathrm{mm}$ from the shell apex.

Savage, 1996, 2002), posean seudoseptalio y deban reasignarse a Iberirhynchiidae o a otros taxones ancistrorincoideos, pero para acreditarlo habría que realizar una revisión detallada de las características internas dorsales de todas las formas de dicha familia, en particular de las menos conocidas, lo que está fuera del alcance del presente trabajo.

A tenor de las edades y distribución paleogeográfica de los géneros discutidos, los Iberirhynchiidae nov. fam. pudieron originarse en el Ordovícico Medio (Darriwiliense), con un tronco basal constituido por el cosmopolita Rostricellula Ulrich \& Cooper, 1942 y los Rostricellulinae Rozman, 1969, del Ordovícico Medio al Silúrico inferior, de los que derivarían en el Ordovícico Superior los Virginiatinae Amsden, 1974, también con amplia distribución paleogeográfica. Más tarde, a lo largo del Silúrico, aparecerían, por una parte, los propios Iberirhynchiinae nov. subfam., siendo Ancillotoechia Havlíček, 1959, una forma cosmopolita del Silúrico medio al Devónico más bajo, su forma más generalizada y, probablemente, ancestral, y una rama 
lateral especializada, de origen oscuro, los Myrmirhynxinae nov. subfam., del Silúrico medio al superior, acantonados en Bohemia (Fig. 8). Los Niorhynicidae Savage, 1996, del Silúrico, con seudoseptalio completo, con o sin conectivo, pero sin placas dentales y con ornamentación radial dividida, podrían constituir un grupo hermano de los Iberirhynchiidae dentro de los Ancistrorhynchoidea o integrar con ellos una nueva unidad superfamiliar (Fig. 8).

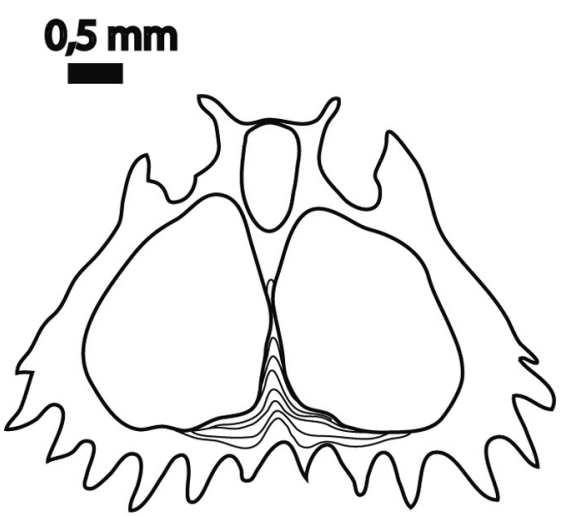

a

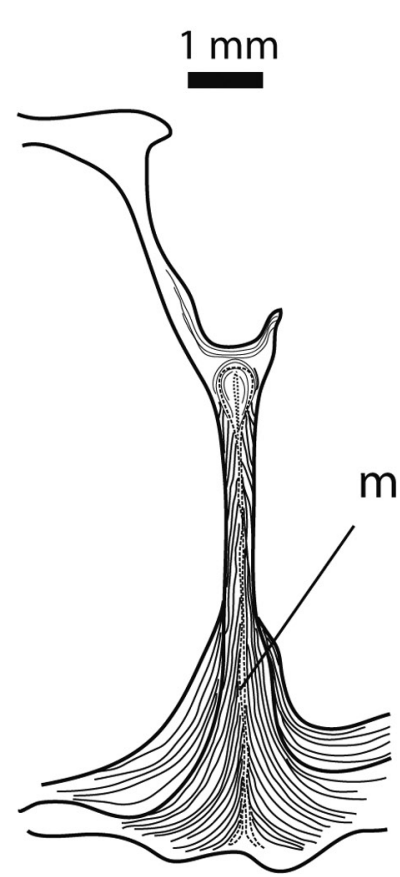

d

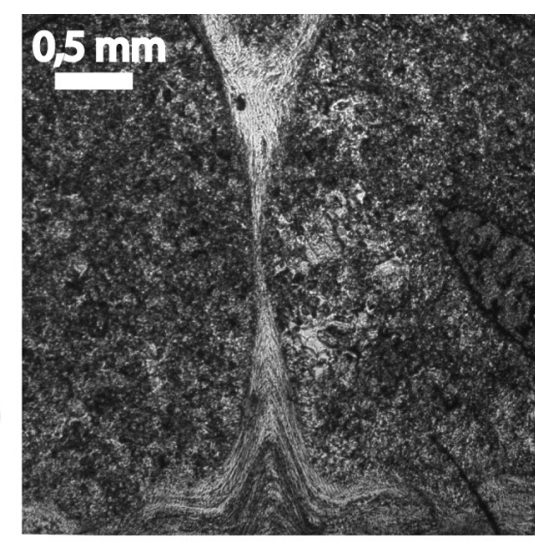

b

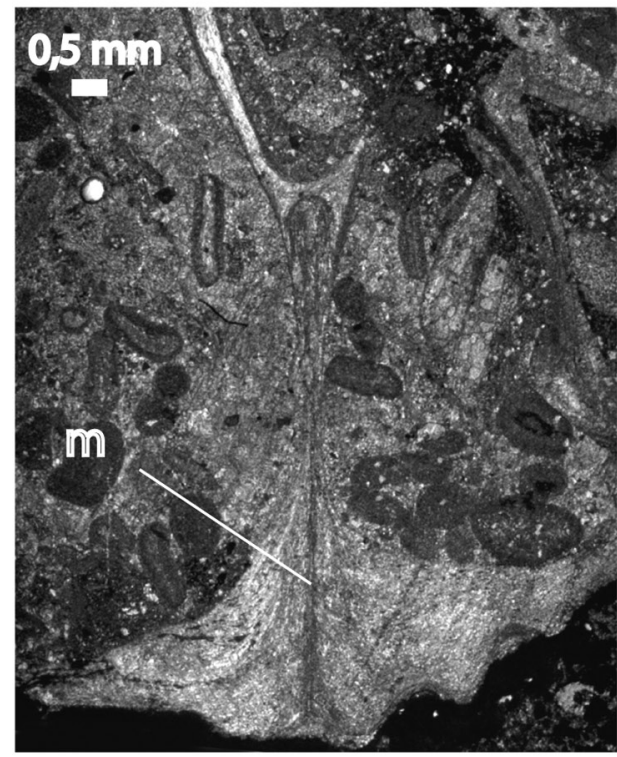

e

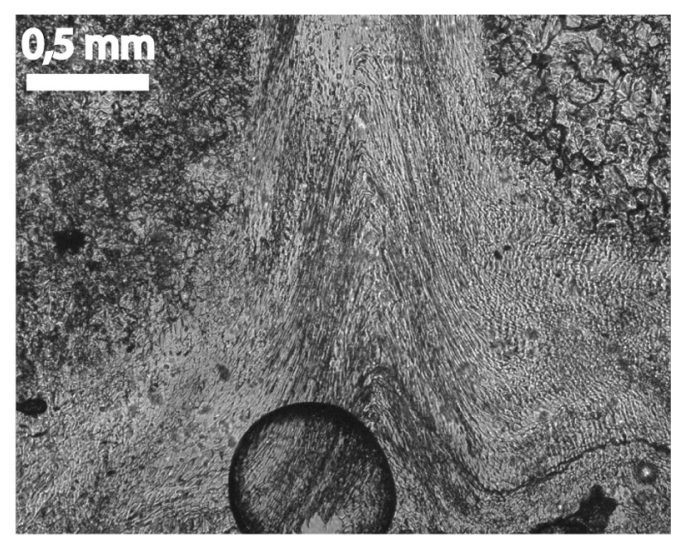

$\mathrm{c}$

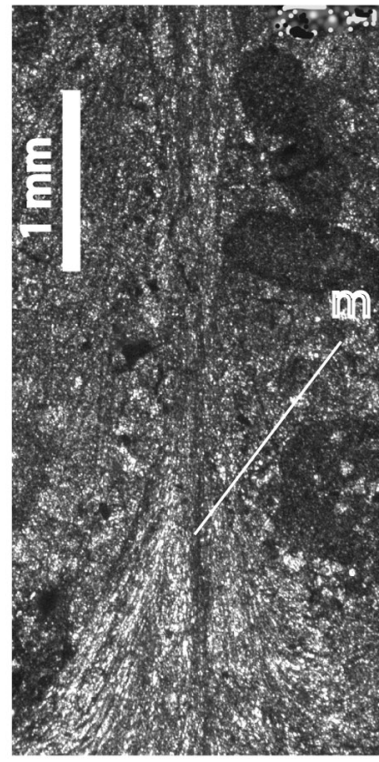

f

Figura 5. Estructura interna del tabique medio dorsal en Iberirhynchia (a-c) y en Trigonirhynchia (d-f). a-c: sección final de la serie representada en figura 4.b del ejemplar DPO 38362-3 de I. santaluciensis: dibujo de la valva dorsal (a), foto algo aumentada del tabique medio (seudosepto) (b) y foto muy aumentada de la base del seudosepto (c). d-f: Réplica al acetato de un ejemplar de Trigonirhynchia fallaciosa (Bayle), de Nehou (Francia), donado por la Dra. Denise Brice: dibujo de la réplica (d), fotografía algo aumentada del septalio (e) y fotografía muy aumentada de la base del septo medio (f). Internal structure of the median dorsal elevation in Iberirhynchia (a-c) and Trigonirhynchia (d-f). a-c: last section of the series represented in figure $4 . b$ of the specimen DPO 38362-3 of I. santaluciensis: draft of the dorsal valve (a), photograph slightly enlarged of the median elevation (pseudoseptum) (b), and photograph strongly enlarged of the base of the pseudoseptum (c).d-f: Acetate peel of a specimen of Trigonirhynchia fallaciosa (Bayle), from Nehou (France), donated by Dr. Denise Brice: draft of the acetate peel $(\boldsymbol{d})$, photograph slightly enlarged of the septalium (e), and photograph strongly enlarged of the base of the median septum $(f)$. 

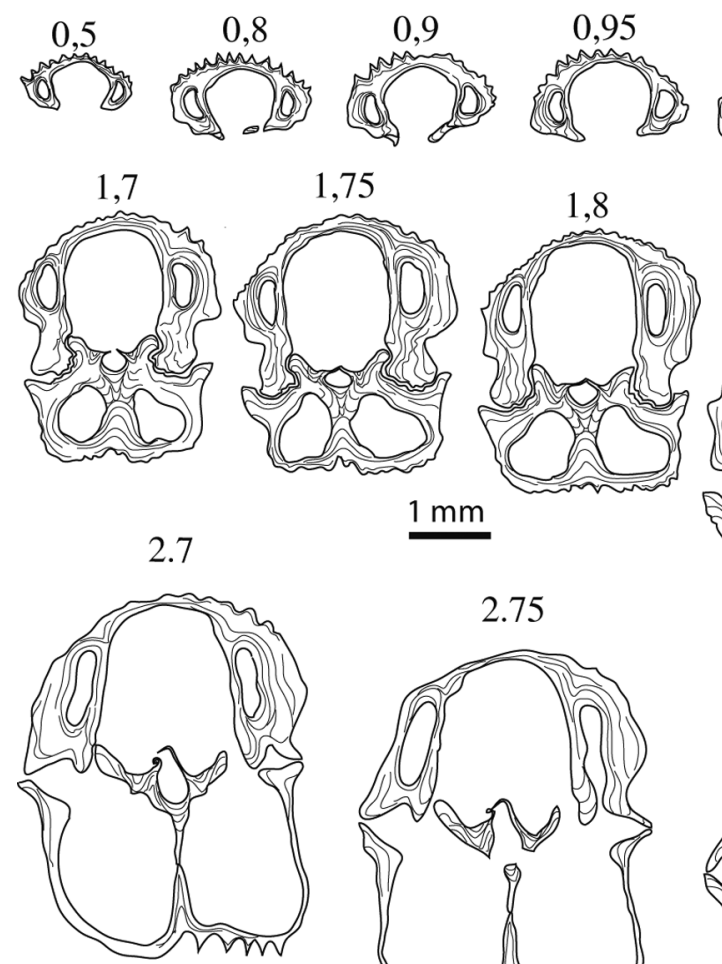

2.75
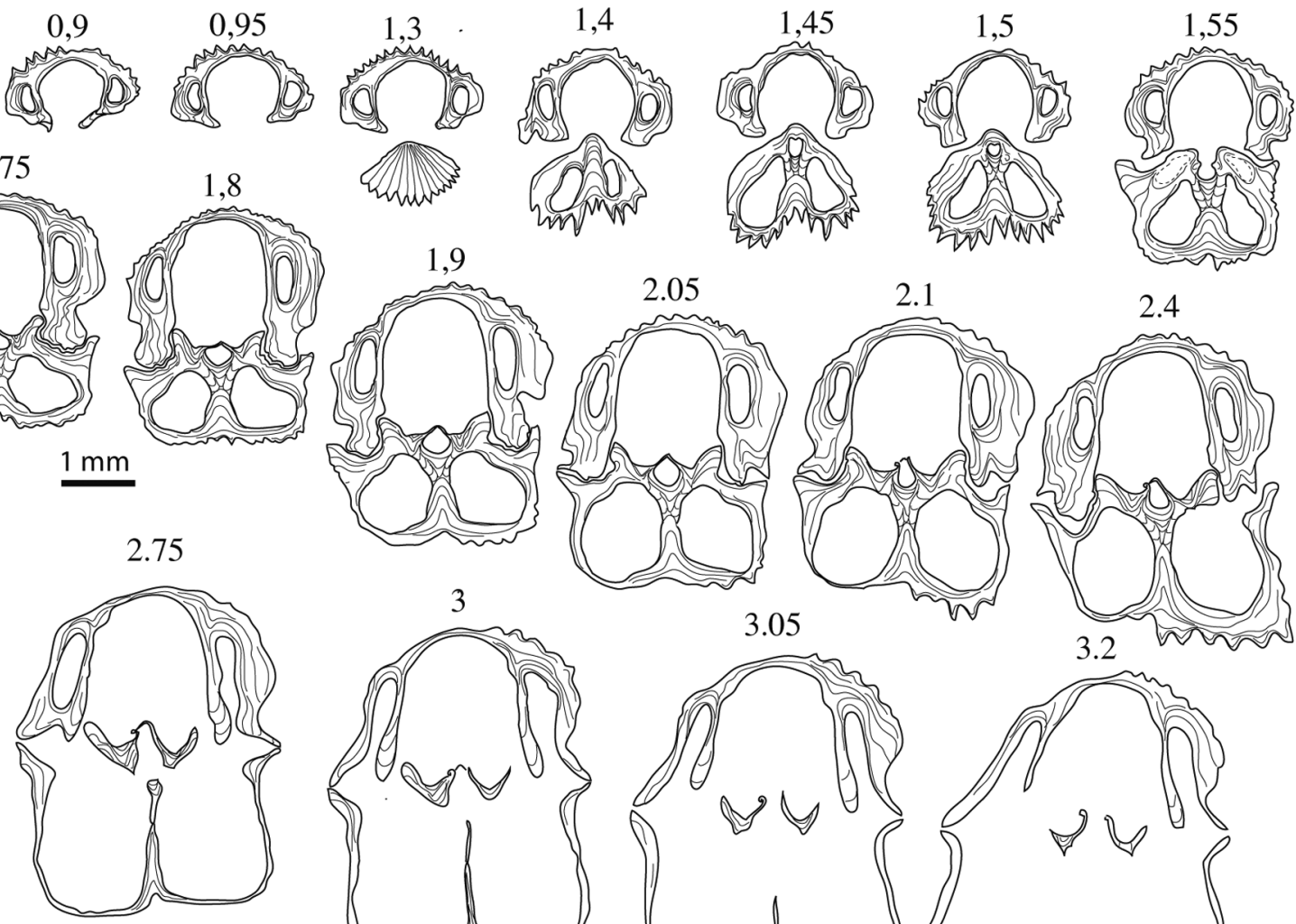

3.3
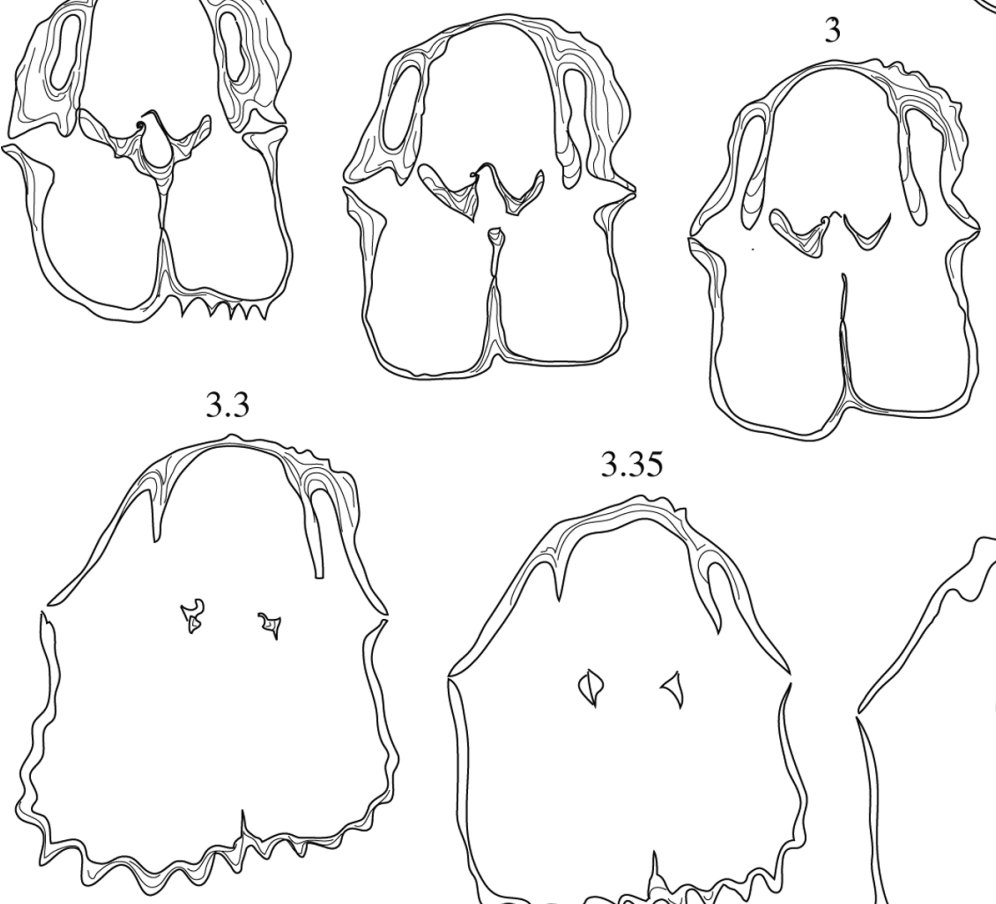

3.35
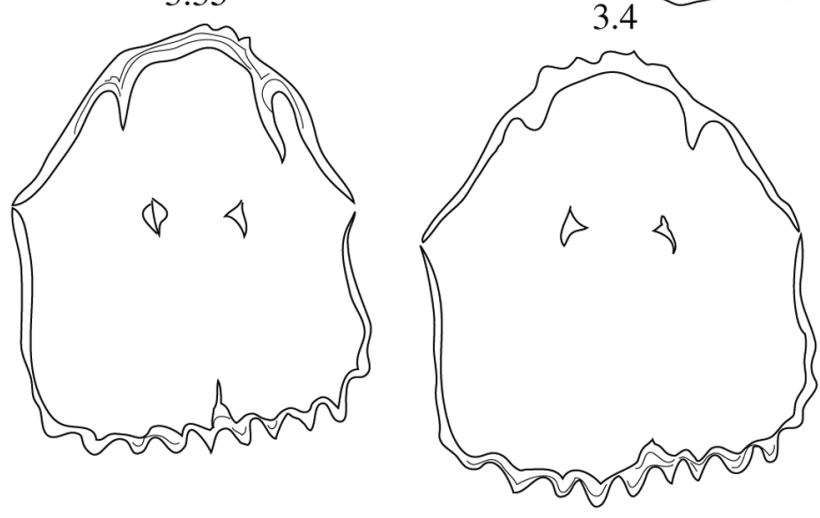

2.1

2.4
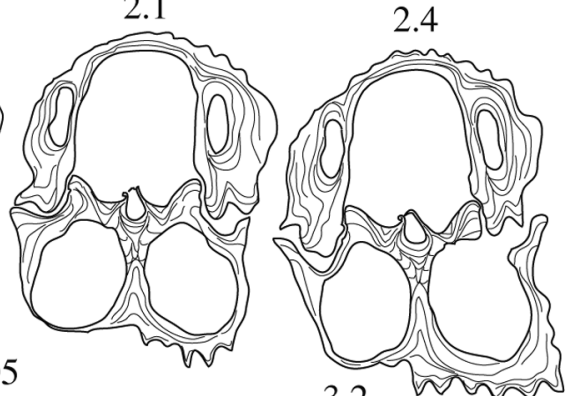

3.2
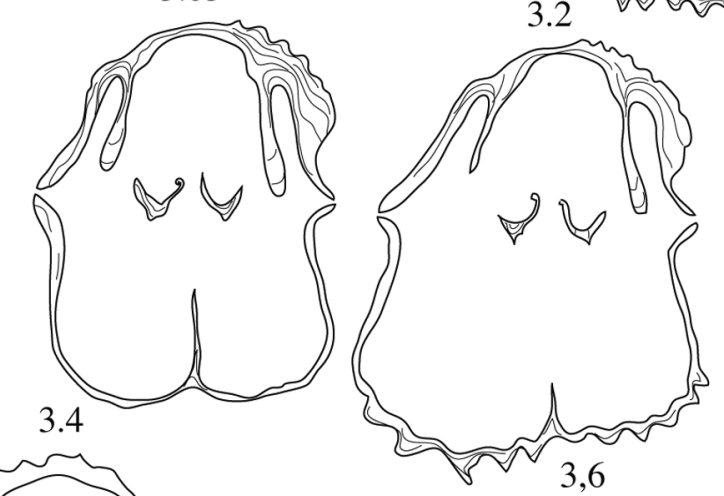

3,9

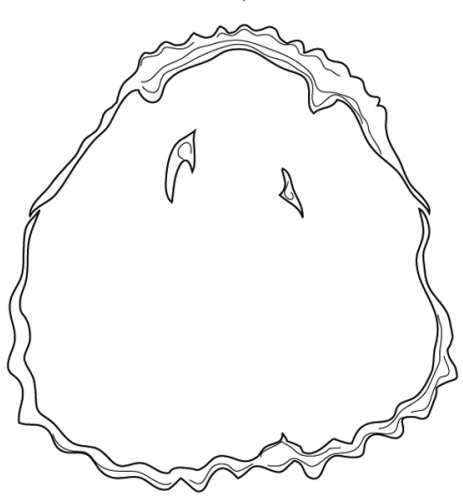

4

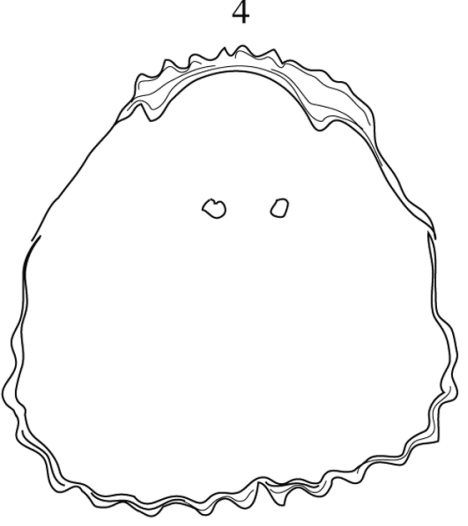

4.4

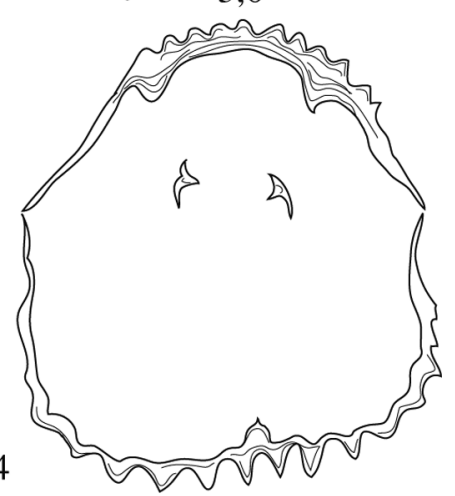

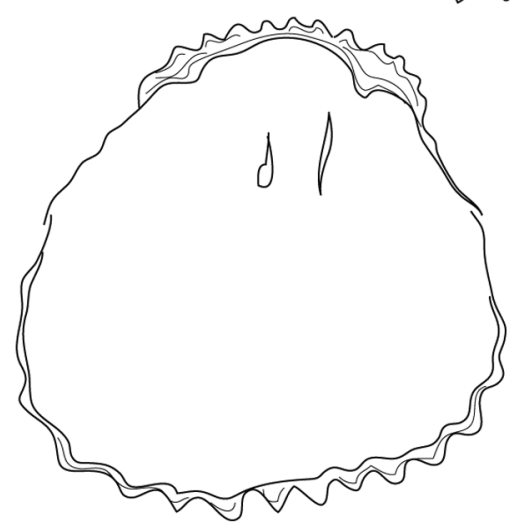

Figura 6. Dibujos con cámara clara de las secciones seriadas de Iberirhynchia santaluciensis Drot \& Westbroek, 1966, DPO $38362-$ $1(\mathrm{~L}=8,9 \mathrm{~mm}, \mathrm{a}=8,5 \mathrm{~mm}, \mathrm{~g}=5,8 \mathrm{~mm})$. Distancias medidas al ápice ventral.

Camera lucida drawings of serial sections of Iberirhynchia santaluciensis Drot \& Westbroek, 1966, DPO 38362-1 (L=8,9 $\mathrm{mm}, w=8,5 \mathrm{~mm}, t=5,8 \mathrm{~mm})$. Distances measured in $\mathrm{mm}$ from the shell apex. 


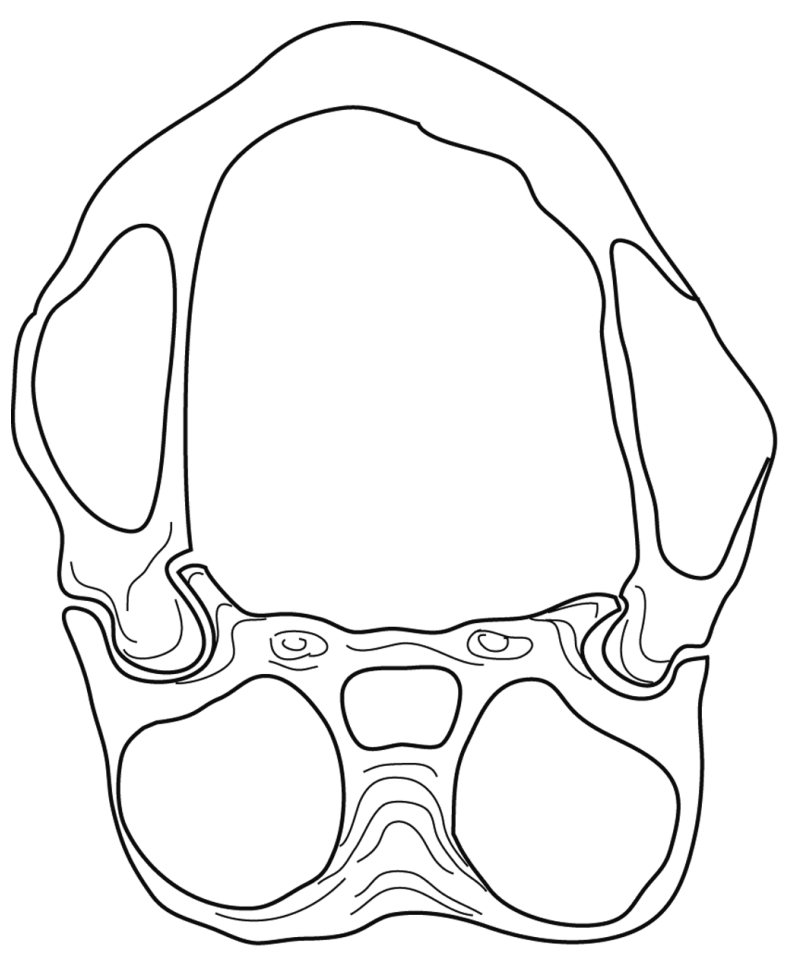

Ancillotoechia

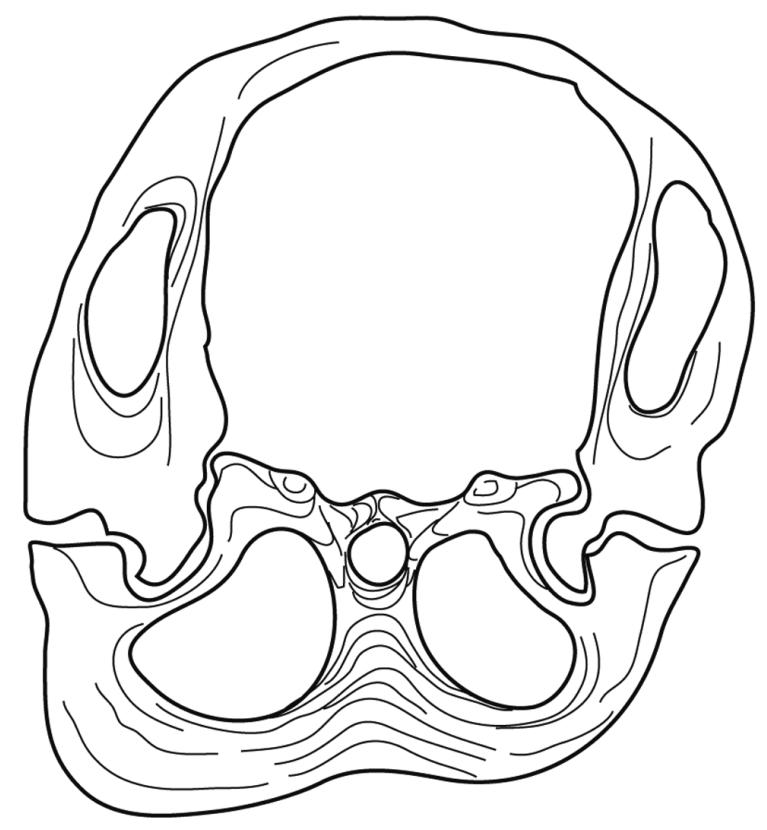

Tectogonotoechia

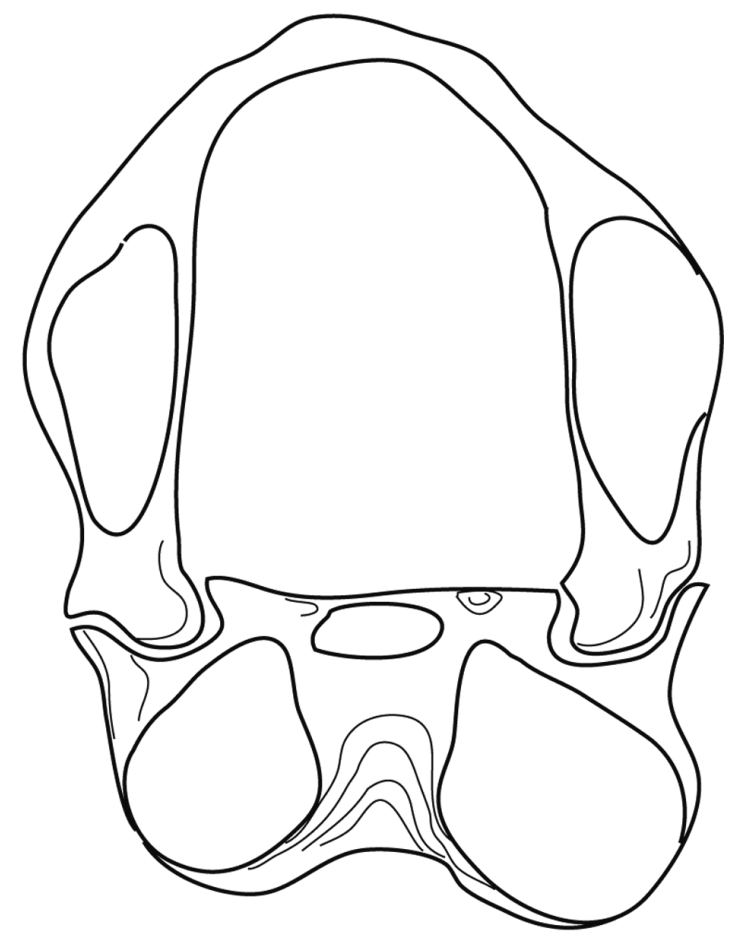

Aratoechia

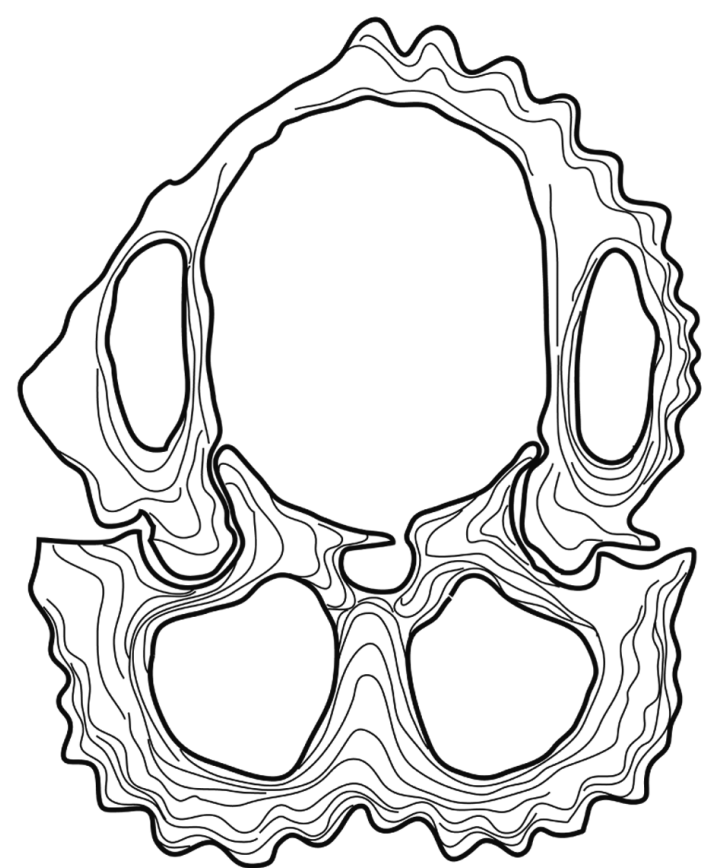

Microsphaeridiorhynchus

Figura 7. Secciones de taxones de Iberirhynchiinae nov. subfam. (no a escala), mostrando la estructura del seudoseptalio. Las de Ancillotoechia Havlíček, 1959, y Aratoechia Havlíček, 1982, redibujadas del trabajo de Havlíček \& Štorch (1990). La de Tectogonotoechia García-Alcalde, 1998, del de García-Alcalde (1998). Y la de Microsphaeridiorhynchus Sartenaer 1970, del de Savage (2002, en Treatise on Invertebrate Paleontology).

Sections of Iberirhynchiinae nov. subfam. taxa (not to scale) showing the pseudoseptalium structure. Sections of Ancillotoechia Havliček, 1959, and Aratoechia Havliček, 1985, redrawn from Havlíček \& Štorch (1990); section of Tectogonotoechia García-Alcalde, 1998, redrawn from García-Alcalde (1998), and that of Microsphaeridiorhynchus Sartenaer, 1970 , redrawn from Savage (2002, in Treatise on Invertebrate Paleontology). 


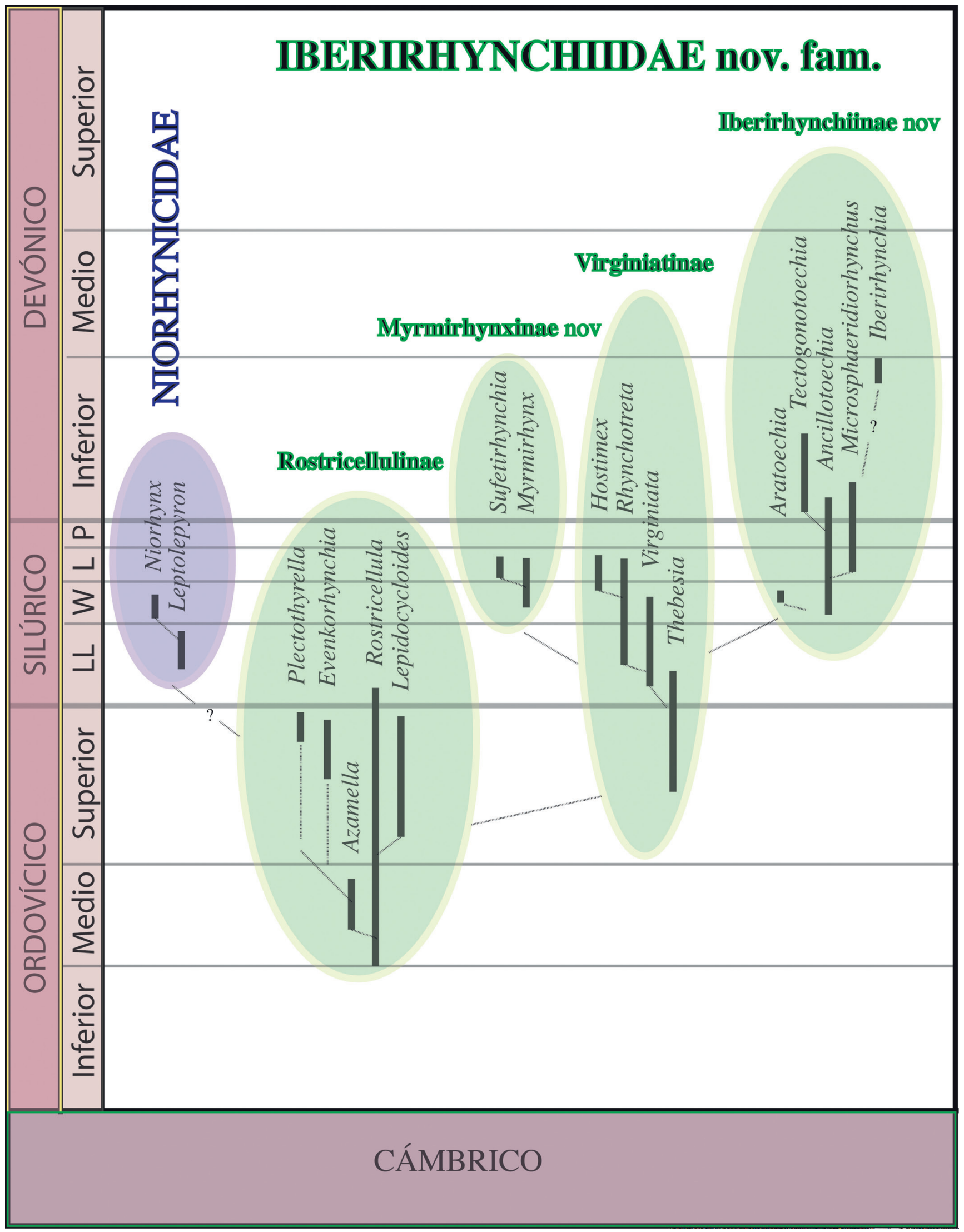

Figura 8. Estratigrafía y filogenia propuesta para los géneros de las familias de Ancistrorhynchoidea Cooper, 1956: Niorhynicidae Savage, 1996 e Iberirhynchiidae nov. fam.

Stratigraphy and proposed phylogeny of the genera of the ancistrorhynchoid families: Niorhynicidae Savage, 1996 and Iberhynchiidae nov. fam. 
La historia y relaciones de Iberirhynchia, el género más moderno de la Subfamilia Iberirhynchiinae (Emsiense superior), con los demás taxones del grupo de Euroamérica y Gondwana y, en particular, con las más próximos en el tiempo, Microsphaeridiorhynchus, del Ludlow al Lochkoviense y Tectogonotoechia, del Lochkoviense al Praguiense, no están claras en el estado actual de conocimientos. La aparente desconexión de las formas del Emsiense superior cantábrico de sus posibles raíces es un hecho intrigante que fue puesto también en evidencia en otros grupos de braquiópodos, por ejemplo, en el espiriférido Boucotiellina García-Alcalde, 2004, el último género conocido de Cyrtinopsidae (García-Alcalde, 2004, 2005). Este aspecto, mal conocido aún, coincide temporalmente, con la reducción y eventual desaparición de barreras oceánicas entre Gondwana y los paleocontinentes septentrionales durante el Devónico Inferior en el curso de la formación de Pangea Menor. El fenómeno pudo conducir a la rápida extinción de los eslabones evolutivos previos inmediatos de Iberirhynchia, en relación con la importante regresión que tuvo lugar hacia la transición Lochkoviense-Praguiense.

La importancia sistemática de Iberirhynchia, como tipo de una nueva unidad familiar, obliga a redescribir su especie-tipo, a fin de aportar datos críticos puestos al día sobre la morfología, semejanzas y diferencias con otros taxones próximos y analizar diferentes problemas relacionados con su posición sistemática, procedencia del material original, elección de los tipos y ontogenia. Dicha labor se basa en la disponibilidad de material propio, probablemente topotípico, abundante y bien situado, así como de ejemplares de otras localidades de las provincias de León y Asturias, y también de una parte de las colecciones originales de la especie, recibida en préstamo del Rijksmuseum van Geologie en Mineralogie, de Leiden, por la eficaz mediación del Profesor Cornelius F. Winkler Prins (17 ejemplares). En total, se han estudiado 250 ejemplares de la especie (medidos y analizados estadísticamente) y otros 200 peor conservados, de los que se tuvieron en cuenta, esporádicamente, aspectos morfológicos concretos, como discontinuidades en el crecimiento y fórmulas costales, y características paleoecológicas, como la presencia, situación y naturaleza de epizoos.

\section{SISTEMÁTICA}

Orden RHYNCHONELLIDA Kuhn, 1949

Superfamilia Ancistrorhynchoidea Cooper, 1956

\section{Familia Iberirhynchiidae nov. fam.}

Género tipo: Iberirhynchia Drot \& Westbroek, 1966.

Diagnosis: Shell small, generally dorsi-biconvex, of subtriangular to triangular outline. Radial costae simple or partially divided, strong, subangular to angular, starting at the valve apexes. Dorsal fold and ventral sinus starting ahead the valve apexes, faint but well defined excepting in the Virginiatinae Amsden, 1974 where both are indistinct. Dorsal umbonal area with a marked median depression that extends distally sometimes as far as to reach the anterior. Dental plates more or less vertical. Dorsal interior with a complete pseudoseptalium, covered or not.

Conchas pequeñas, generalmente dorsibiconvexas, de contorno subtriangular a triangular, con pliegues fuertes, subangulosos a angulosos, iniciándose en los ápices de las valvas, simples o, en parte, divididos. Pliegue medio dorsal y seno medio ventral débiles pero generalmente bien individualizados, salvo en los Virginiatinae Amsden, 1974, naciendo por delante de los ápices. Región umbonal dorsal deprimida medianamente, depresión que puede extenderse hacia delante incluso a lo largo de toda la valva. Placas dentales más o menos verticales. Interior dorsal con seudoseptalio completo, cubierto o no por un conectivo.

Discusión: Como ya se indicó antes, varios taxones integrados hasta ahora en la Familia Trigonirhynchiidae Schmidt, 1965, tienen el tabique medio dorsal constituido exclusivamente por concha secundaria (seudosepto), formando con las placas crurales un seudoseptalio (sensu Rozman, 1969, fide Havlíček \& Štorch, 1990). Siguiendo en parte a Havlíček \& Štorch (1990), se considera aquí, como ya se expuso previamente, dicho carácter determinante para separar todas las formas que lo presentan, no sólo de Trigonirhynchiidae sino también de la Superfamilia Rhynchotrematoidea Schuchert, 1913, y reasignarlas provisionalmente a Ancistrorhynchoidea Cooper, 1956. Por otro lado, las formas seudoseptaliadas, con placas dentales, se asignan a la Familia Iberirhynchiidae nov. fam., compuesta por las subfamilias Iberirhynchiinae nov. subfam., Myrmirhynxinae nov. subfam., Rostricellulinae Rozman, 1969, y Virginiatinae Amsden, 1974. Las características diagnósticas de estos taxones, su composición genérica, relaciones recíprocas y filogenia se comentaron antes, en el epígrafe "Esquema clasificatorio".

Edad, paleobiogeografía y filogenia: Los taxones de la Familia Iberirhynchiidae nov. fam. se distribuyen del Ordovícico Medio a la parte alta del Devónico Inferior (Fig. 8). La gran mayoría de ellos son propios de los dominios paleogeográficos de Euroamérica y Gondwana septentrional, pero algunos (datos pendientes de confirmación) fueron citados en dominios diferentes (ver Savage, 2002, in Treatise on Invertebrate Paleontology). La familia derivaría de alguna forma próxima a los Virginiatinae, con seudoseptalio prácticamente cubierto y con seno ventral y pliegue medio dorsal presentes.

\section{Subfamilia Iberirhynchiinae nov. subfam.}

Género tipo: Iberirhynchia Drot \& Westbroek, 1966. 
Diagnosis: Iberirhynchiidae of subtriangular to triangular outline. Median umbonal depression more or less extended forward, sometimes reaching the anterior commissure. Radial costae rather simple. Dental plates vertical or nearly so. Seudoseptalium covered.

Iberirhynchiidae de contorno subtriangular a triangular, depresión umbonal más o menos importante, extendiéndose a veces hasta la propia comisura frontal; costillas generalmente simples; placas dentales verticales o casi y seudoseptalio completo cubierto por un conectivo de forma variable.

Géneros asignados: Además del tipo, se incluyen en esta subfamilia Ancillotoechia Havlíček, 1959, Microsphaeridiorhynchus Sartenaer, 1970, Aratoechia Havlíček, 1982, y Tectogonotoechia García-Alcalde, 1998.

Edad y filogenia: Los taxones de la subfamilia se distribuyen del Silúrico al Devónico Inferior (WenlockEmsiense superior) (Fig. 8). Como ya se indicó antes, la forma ancestral del grupo podría ser el género cosmopolita Ancillotoechia, del que derivaría en el Wenlock Aratoechia, con tendencia a la división de las costillas radiales, y más tarde, en el Ludlow, Microsphaeridiorhynchus, y en el Lochkoviense superior, Tectogonotoechia, ambos con ornamentación radial simple. Como también se señaló en su momento, el origen del género más moderno del grupo, Iberirhynchia, no está claro y no contribuye a aclarar la situación el intervalo temporal de varios millones de años que lo separa del más moderno de los restantes géneros de la subfamilia (Fig. 8). El argumento paleogeográfico lo enlazaría con Tectogonotoechia, pero se aproxima morfológicamente más a $M i$ crosphaeridiorhynchus.

\section{Género Iberirhynchia Drot \& Westbroek, 1966}

Especie-tipo: Iberirhynchia santaluciensis Drot \& Westbroek, 1966.

Discusión: La diagnosis original del género en Drot \& Westbroek (1966, p. 165) es bastante ilustrativa y requiere escasos retoques, salvo los referidos a la posesión de seudoseptalio en el interior de la valva dorsal y la prolongación de la depresión umbonal media de la valva dorsal hasta la misma comisura anterior.
Procedencia, situación estratigráfica y edad: El género sólo se conoce, hasta el presente, en el dominio AsturLeonés de la Cordillera Cantábrica (provincias de Asturias y León, $\mathrm{N}$ de España), en la parte alta de las formaciones Santa Lucía y Moniello, particularmente en el Intervalo Faunístico 16 (García-Alcalde, 1996). Esta situación correspondería, de acuerdo con los datos disponibles, a la zona de conodontos Icriodus costatus patulus, de finales del Emsiense superior (Fig. 9). Las referencias al género en Bohemia (Havlíček \& Kukal, 1990: Iberirhynchia nargis, y Havlíček, 1992: Iberirhynchia sp.) corresponden a verdaderos Trigonirhynchiidae, con septalio carente de conectivo y septo medio dorsal convencional, con mediotest y concha secundaria, por lo que se rechazan del género Iberirhynchia, que estaría por el momento compuesto sólo por su especie-tipo.

\section{Iberirhynchia santaluciensis Drot \& Westbroek, 1966}

Figuras 1-6, 12-13

v. 1966 Iberirhynchia santaluciensis Drot et Westbroek n.gen. n.sp.; Drot \& Westbroek, p. 167.

v. 1990 Iberirhynchia santaluciensis; García-Alcalde en Truyols et al, Fig. 2.

v. 1995 Iberirhynchia santaluciensis; García-Alcalde, Fig. 6.

v. 1996 Iberirhynchia santaluciensis; García-Alcalde, Fig. 2.

v. 2001 Iberirhynchia santaluciensis; García-Alcalde, Fig. 2.

v. 2006 Iberirhynchia santaluciensis; García-Alcalde, en Ellwood et al, Fig. 2.

Holotipo: Ejemplar St. 114509, depositado en el Rijksmuseum van Geologie en Mineralogie, de Leiden (Holanda), procedente del área del Pico Aguasalio (cerca de Crémenes, valle del río Esla).

Material: Hay diversos aspectos cuestionables o poco claros relacionados con el material original de I. santaluciensis.

a) Procedencia del holotipo. En la publicación original (Drot \& Westbroek, 1966, p. 167) no hay indicación precisa de la procedencia del holotipo. Respecto al material de estudio, se citan, al menos, dos localidades diferentes: Pico Aguasalio y valle del río Bernesga. Del Pico Aguasalio se manejan las colecciones de Mme. F.A. Hofsteede de Groot-Sprenger van Eyjk, compuesta por 230 paratipos, y la de M. P.F.L. de Groot, con 129 ejemplares. Y del valle del río Bernesga, cerca de Huergas, una colección, la de G.C. Cadée, con 17 ejemplares. Podría suponerse

Figura 9. Columna estratigráfica de la supuesta localidad-tipo de Iberirhynchia santaluciensis Drot \& Westbroek, 1966, en la pista a lo largo del arroyo de La Trapa, vertiente occidental de Peña Escricia, 500 m al ESE de Argovejo, provincia de León. El estrato típico correspondería al nivel AR-SL-5 de la figura, en la parte alta del Miembro III y del Intervalo Faunístico 16 de la Formación Santa Lucía, Emsiense terminal.

Stratigraphical column of the supposed Iberirhynchia santaluciensis Drot \& Westbroek, 1966, locus typicus. Track along the La Trapa creek, western slope of Peña Escricia, $500 \mathrm{~m}$ ESE of Argovejo, province of León. The stratum typicum would correspond to the bed AR-SL-5 in the upper part of the Member III and of the Faunal Interval 16, Santa Lucía Formation, latest Emsian. 


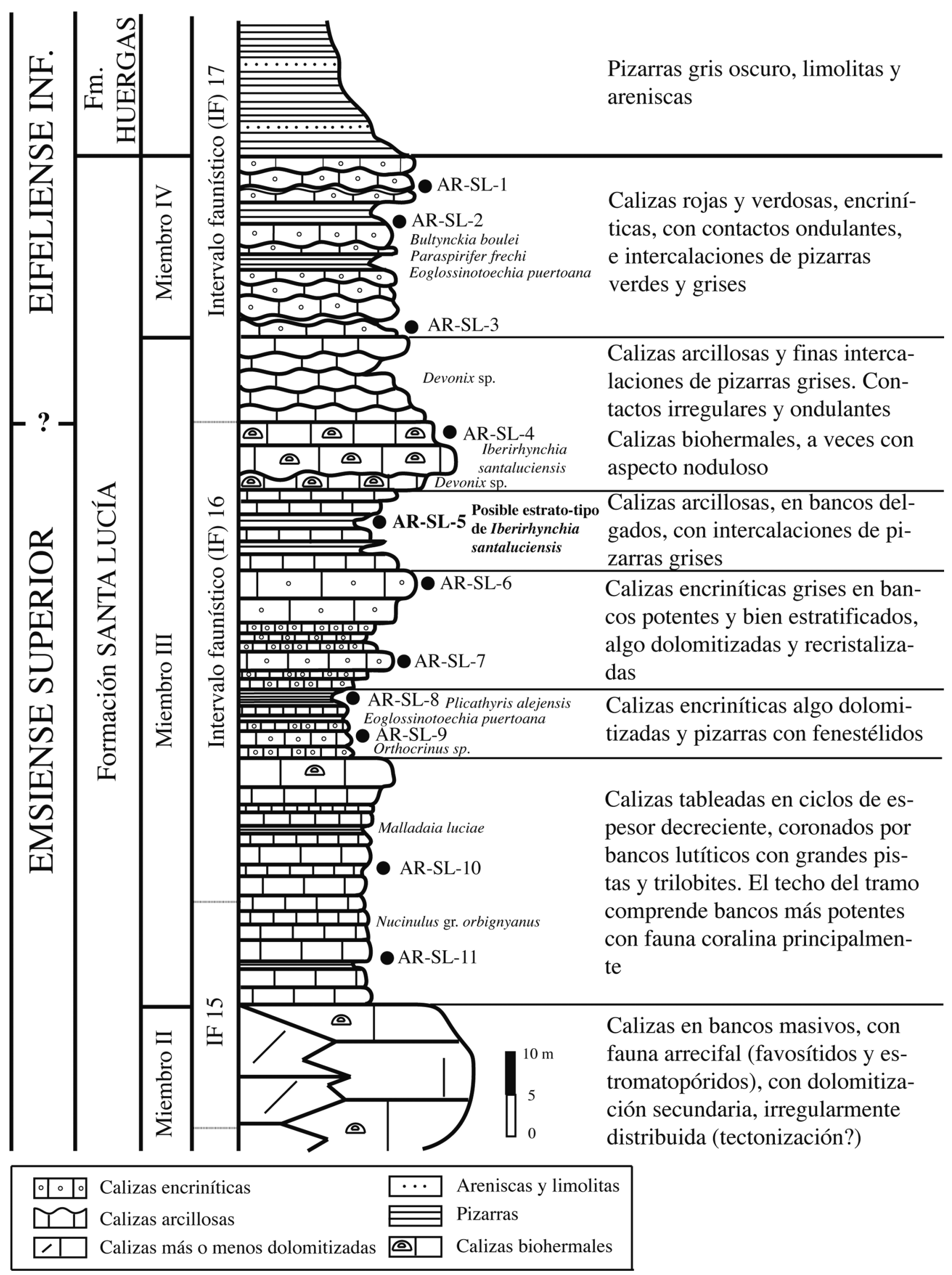


que el holotipo viene de la primera de las colecciones del Pico Aguasalio pero habría que subrayarlo inequívocamente.

b) Numeración de los ejemplares. Se citan los números St. 114509 a 114527 de la colección del Rijksmuseum van Geologie en Mineralogie de Leiden, como registro del material estudiado, pero no se aclara la correspondencia concreta de los números con cada una de las colecciones y únicamente se sabe que el holotipo, de procedencia imprecisa, tiene la sigla St. 114509.

c) Designación y numeración de los paratipos y localización actual del material tipo. En principio, los paratipos escogidos por los autores fueron 230 ejemplares de la colección de Mme. F.A. Hofsteede de Groot-Sprenger van Eyjk, cuya numeración precisa no se conoce, de los cuales, 117 se conservarían " $a$ l'Institut de Paléontologie du Muséum nacional d'Histoire naturelle de Paris, sous le numéro provisoire 1965-14"; pero los ejemplares figurados, aparte del holotipo, con siglas St. 114510 a 114514, no consta qué estatuto tienen, aunque algunos sean de la localidad y estrato típicos (podrían ser de la colección de Mme. F.A. Hofsteede o de la de M. P.F.L. de Groot). Tampoco se conoce la procedencia y el estatuto de los tres ejemplares cuyas medidas se proporcionan junto con las del holotipo. Finalmente, se ignora qué números originales o definitivos tendrían los paratipos trasladados a Paris y si aún continúan en aquella ciudad.

d) Situación precisa de la localidad y estrato típico. La localidad típica, designada como pico de Aguasalio (cerca de Crémenes, valle del Esla), es una referencia incompleta, teniendo en cuenta que se trata de un área bastante extensa, con numerosos afloramientos de la Formación Santa Lucía. La capa V 28 de Mme. F.A. Hofsteede de Groot-Sprenger van Eyjk, que consta como estrato típico, no se conoce a ciencia cierta a cual de los cortes posibles de la Formación Santa Lucía pertenece ni su posición estratigráfica dentro de la formación, a falta de mapas y columnas estratigráficas detalladas que debían de figurar en el trabajo original (inédito) y que no hemos podido conseguir, pese a múltiples gestiones en Holanda, Madrid, La Coruña y Oviedo. Sin embargo, teniendo en cuenta lo abundante del muestreo y el tipo de fosilización, es muy posible que la localidad-tipo se encuentre en el sendero a lo largo del arroyo de La Trapa que, más abajo, se describe.

e) Naturaleza del holotipo y otros ejemplares medidos. El holotipo es un ejemplar de $10 \mathrm{~mm}$ de longitud, 11 de anchura y 7,1 de grosor. Estas medidas corresponden a un ejemplar muy maduro, incluso geróntico, que no expresa demasiado bien las características más generales de la especie. Y lo mismo sucede con los otros tres ejemplares medidos, que superan también con claridad las medias de longitud, anchura y grosor de nuestro propio material. En efecto, de los cerca de 250 ejemplares medidos de nuestras colecciones, sólo 13 superan o igualan la longitud del holotipo, 26 la anchura y 39 el grosor. La figuración de otros ejemplares en diferentes estadios de desarrollo en la publicación original palía, en parte, el problema y permite una mejor comprensión de la especie.

En el presente trabajo se utilizó una pequeña parte del material original y numerosos ejemplares propios de las siguientes procedencias y con los números de colección que se indican:
A) Material original depositado en el Rijksmuseum van Geologie en Mineralogie, de Leiden:

a) 1 ejemplar (juvenil, efébico precoz), RGM 114510, procedente de la localidad tipo, nivel V28, Pico Aguasalio, Formación Santa Lucía, colección F.A. Hofsteede de Groot-Sprenger van Eyjk; figurado en Drot \& Westbroek, 1966, pl. 1, fig. 4.

b) 1 ejemplar (juvenil, efébico precoz), RGM 114514, de la localidad 19, caliza de Santa Lucía, Huergas de Gordón, de la colección de G.C. Cadée; figurado en Drot \& Westbroek, 1966, pl. 1, fig. 6).

c) 10 ejemplares (principalmente adultos), RGM 114515, procedentes de la localidad tipo, nivel V28, Pico Aguasalio, Formación Santa Lucía, colección F.A. Hofsteede de Groot-Sprenger van Eyjk.

d) 3 ejemplares (en mal estado; un adulto, un juvenil y el tercero perteneciente a un género completamente distinto de Retzoidea), RGM 114519, procedente, con dudas, del área del Pico Aguasalio, nivel CL22 de la col. de P.F.L. de Groot.

e) 2 ejemplares adultos, en regular estado, RGM 114523, de las proximidades de la localidad tipo, nivel V29, de la col. F.A. Hofsteede de Groot-Sprenger van Eyjk.

B) Material propio, depositado en el Museo de Geología de la Universidad de Oviedo:

a) 374 ejemplares. 227 de ellos, DPO 38361 (1-227), en buen estado (Figs. 1-4, 12-13) y otros 147 en mal estado, DPO 38362 (1-147) (Fig. 6) procedentes de la pista a lo largo del arroyo de La Trapa, unos $500 \mathrm{~m}$ al ESE del pueblo de Argovejo (Crémenes, Cistierna, provincia de León, N de España) (Fig. 10).

b) 1 ejemplar en regular estado, DPO 39552 procedente de la misma localidad anterior, pero de un nivel diferente, algo más antiguo.

c) 9 ejemplares, DPO 38496 (1-9), en variable estado de conservación, procedentes de la prolongación occidental del estrato tipo, en la margen izquierda del arroyo de La Trapa.

d) 18 ejemplares de El Millar (Huergas de Gordón, León), DPO 39553-39570. La colección de M G.C. Cadée, del valle del río Bernesga, cerca de Huergas, a que se alude en el trabajo original, podría proceder de esta misma localidad.

e) 2 ejemplares, en regular estado, DPO 39527-39528, de Ciñera, valle del río Bernesga, provincia de León.

f) 3 ejemplares silicificados, DPO 39529, 39539-39540, de la localidad de Arnao, Castrillón, provincia de Asturias.

g) 3 ejemplares silicificados, DPO 39530-39532, de una localidad próxima a la anterior, en la playa de El Cuerno.

h) 11 ejemplares silicificados, DPO 39541-39551 del Mirador de Salinas, Avilés, provincia de Asturias.

i) 6 ejemplares silicificados, DPO 39533-39538, de la localidad de Piedras Blancas, Castrillón, provincia de Asturias.

Localidad y estrato típico: La mayor parte de los problemas relacionados con el material tipo no pudieron resolverse hasta ahora, al no conseguir las notas originales y tesinas de los investigadores que reunieron el material ni del único superviviente de los que lo describieron por primera vez, el Dr. Peter Westbroek. Sin embargo, a partir de los datos disponibles, la localidad típica parece ser, como ya se avanzó más arriba, la pista a lo largo del arroyo de La Trapa, en la vertiente occidental de Peña Escricia, unos $500 \mathrm{~m}$ al ESE del pueblo de Argovejo (Crémenes, Cistierna, provincia de León, $\mathrm{N}$ de España) (Figs. 10-11) y el estrato típico, calizas arcillosas decimétricas, de color gris oscu- 
ro, con finas intercalaciones pizarrosas, pertenecientes a la parte superior del Miembro III de la Formación Santa Lucía, nivel AR-SL-5 del Emsiense final, techo del Intervalo Faunístico 16 (García-Alcalde, 1996) (Fig. 9). Esta localidad sería la misma que la loc. 25 de Schumann (1965, P1. 6).
Descripción: Concha distrófica, pequeña (L entre 4 y $11 \mathrm{~mm}$ ), más ancha que larga (media a/L: 1,12), dorsibiconvexa y globosa (media g/L: 0,68), de contorno subtriangular a subpentagonal, a veces débilmente emarginado en el frente, con la máxima anchura en el tercio anterior de la longitud (media: 71\%L); máxima

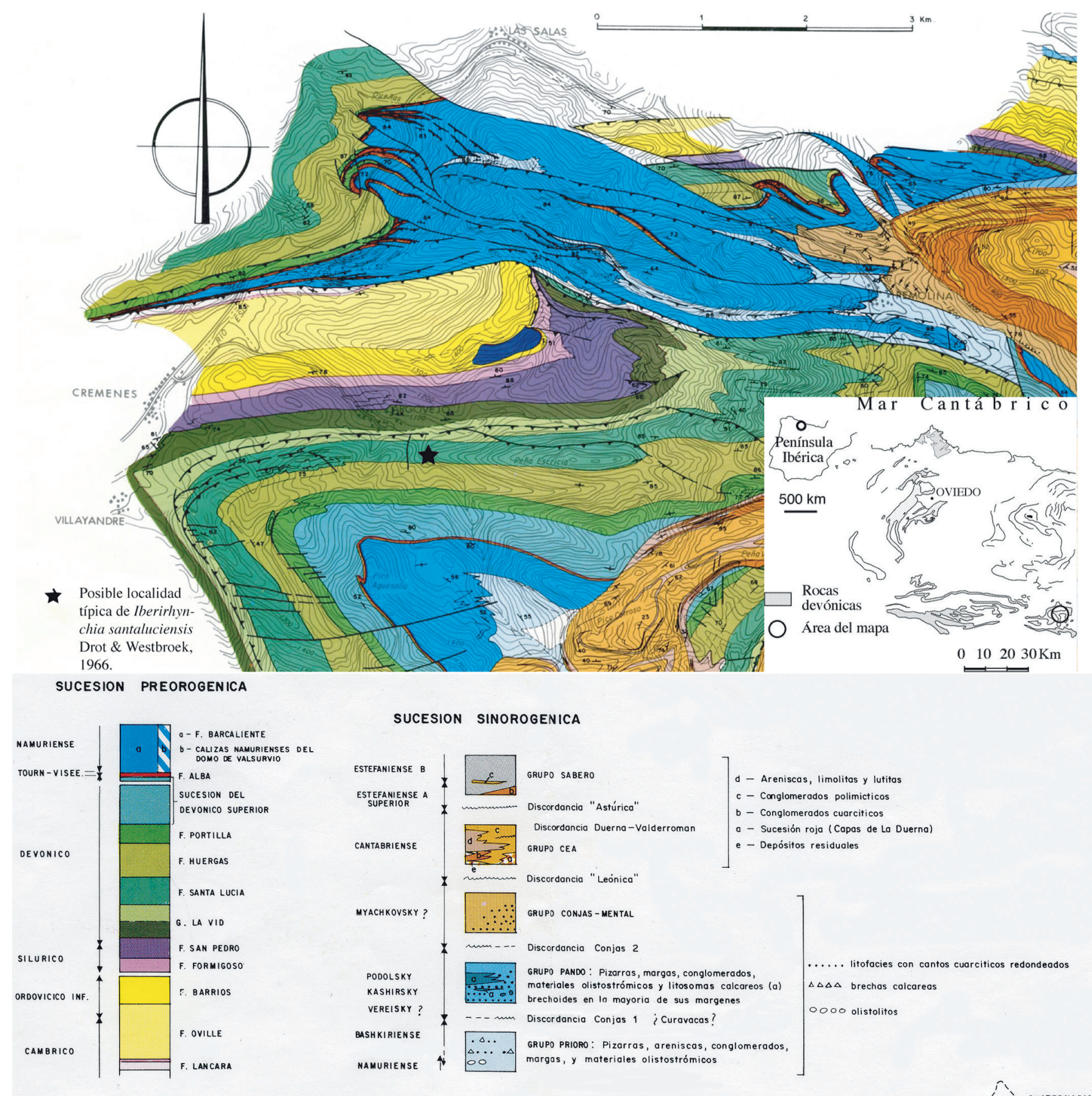

Y cuaternakio

Figura 10. Situación geográfica y geológica de la supuesta localidad-tipo de Iberirhynchia santaluciensis Drot \& Westbroek, 1966, en el flanco Sur del sinclinal de Aguasalio, vertiente occidental de Peña Escricia, $500 \mathrm{~m}$ ESE Argovejo, provincia de León. (Tomado de Alonso, 1985).

Geographic and geologic setting of the supposed Iberirhynchia santaluciensis Drot \& Westbroek, 1966, locus typicus, southern flank of the Aguasalio syncline, western slope of Peña Escricia, $500 \mathrm{~m}$ ESE of Argovejo, province of Leon. (From Alonso, 1985). 
altura en el frente de la concha o algo detrás. Palintropo alto y cóncavo, limitado por aristas umbonales agudas, dividido por una abertura peduncular, mesotírida a permesotírida, prolongándose en una escotadura deltirial limitada basalmente por placas deltidiales separadas. Gancho puntiagudo y estrecho, inclinado a suberecto; ángulo apical agudo (media: $62^{\circ}$ ). Valvas acusadamente convexas en sentido longitudinal; en sentido transversal, la parte media es suavemente convexa, pero los flancos y la región anterior se vuelven abruptamente hacia la comisura encontrándose en ángulos próximos a $180^{\circ}$ en los flancos y algo menos en el frente; comisuras fuertemente indentadas (Figs. 1.c4, 2.c4), pero sin espinas marginales; comisura anterior uniplegada, con lengüeta trapezoidal alta, dirigida anterodorsal a posterodorsalmente (Fig. 2); comisuras laterales prácticamente rectas, en ocasiones algo desviadas en dirección ventral, bisecando sendas lúnulas ovaladas y lisas, bien desarrolladas en los flancos umbonales (Fig. 2.b4). Seno ventral y pliegue medio dorsal muy débiles, iniciándose hacia la mitad anterior de la concha, definidos con claridad sólo cerca de la base de la lengüeta; seno ocupando en el frente por término medio el $66 \%$ de la anchura, de fondo aplanado a débilmente convexo; pliegue medio dorsal deprimido en su parte media, sobre todo umbonalmente (Fig.1.c2,1.c4). Concha cubierta de costillas radiales simples y rectas (un solo ejemplar mostraba un elemento bifurcante), en número de 24 a 36 , que se inician en los ápices de las valvas, subangulosas a angulosas, bastante altas, separadas por espacios intercostales similares a las costillas; la fórmula costal, en la parte media, varía entre 5/4 y 9/8, pero la fórmula 6/5 la presentan más del $80 \%$ de los ejemplares. Las costillas que limitan el pliegue dorsal y el seno ventral suelen ser más fuertes que las otras; en el pliegue medio dorsal, el par lateral o, más frecuentemente, el sublateral (en los ejemplares mejor conservados) destacan algo en altura del resto (Fig. 1.e3, 2.c4); en el seno ventral, el par lateral tiende, en ocasiones, a ocupar un nivel inferior a las vecinas de los flancos. Líneas de crecimiento muy finas y próximas. Una, dos o, más rara vez, tres de las lamelas de crecimiento están muy

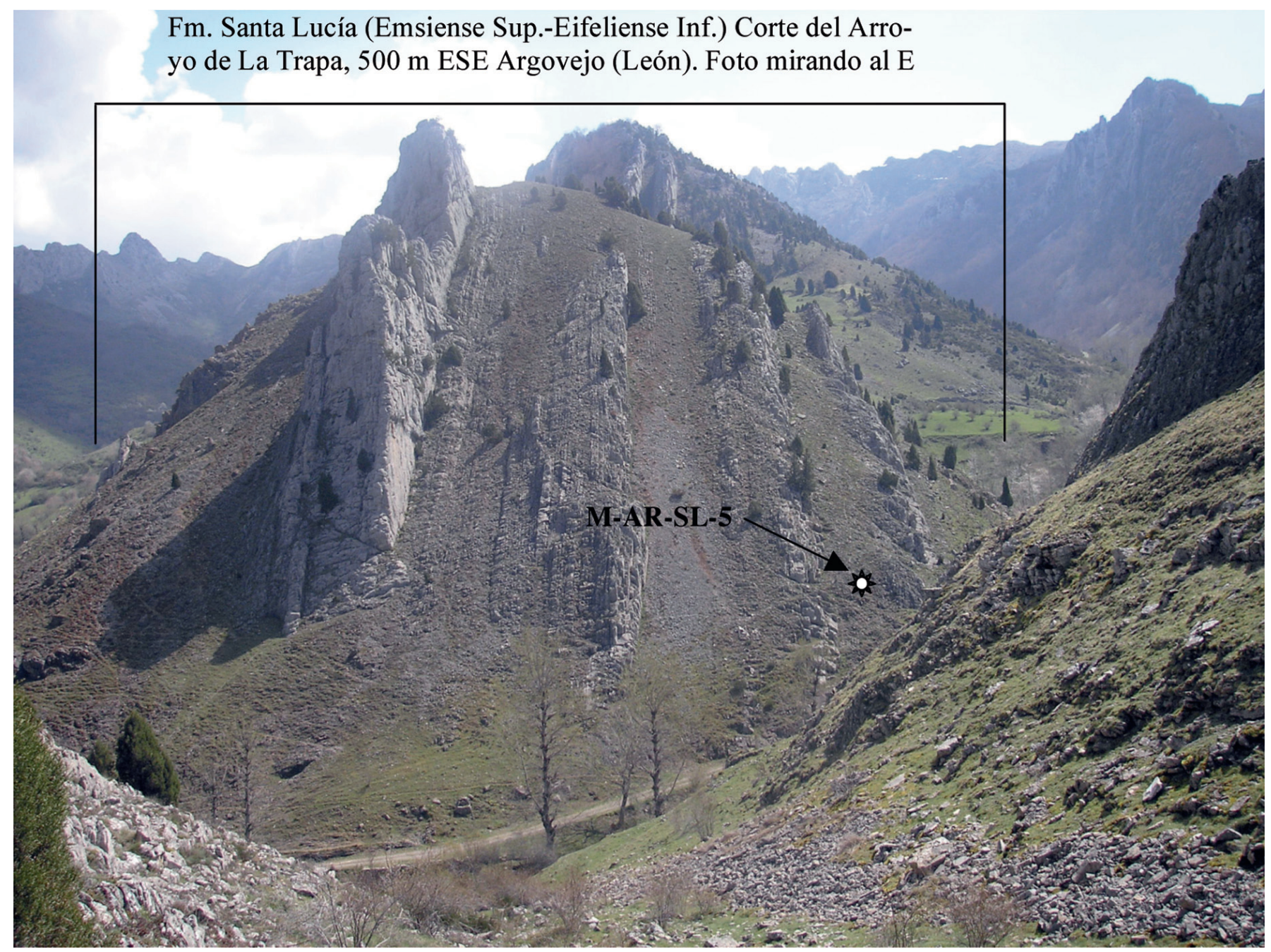

Figura 11. Panorámica mirando al Este, mostrando el corte geológico de la Formación Santa Lucía, en Peña Escricia, ESE de Argovejo, provincia de León y la situación del supuesto estrato típico de Iberirhynchia santaluciensis Drot \& Westbroek, 1966 (nivel AR-SL-5).

Panoramic view looking eastern, showing the Santa Lucia Formation geologic section in Peña Escricia, ESE of Argovejo, province of Leon, and the supposed Iberirhynchia santaluciensis Drot \& Westbroek, 1966, stratum typicum (bed AR-SL-5). 
engrosadas, ilustrando detenciones en el crecimiento, que parecen relacionadas con las etapas principales de desarrollo de la concha (Fig. 13), como se detalla luego; con cierta frecuencia, en dichos niveles se alteran los vectores de crecimiento, produciéndose desviaciones en la dirección de crecimiento de las costillas, flexiones de las mismas, aparición o desaparición de
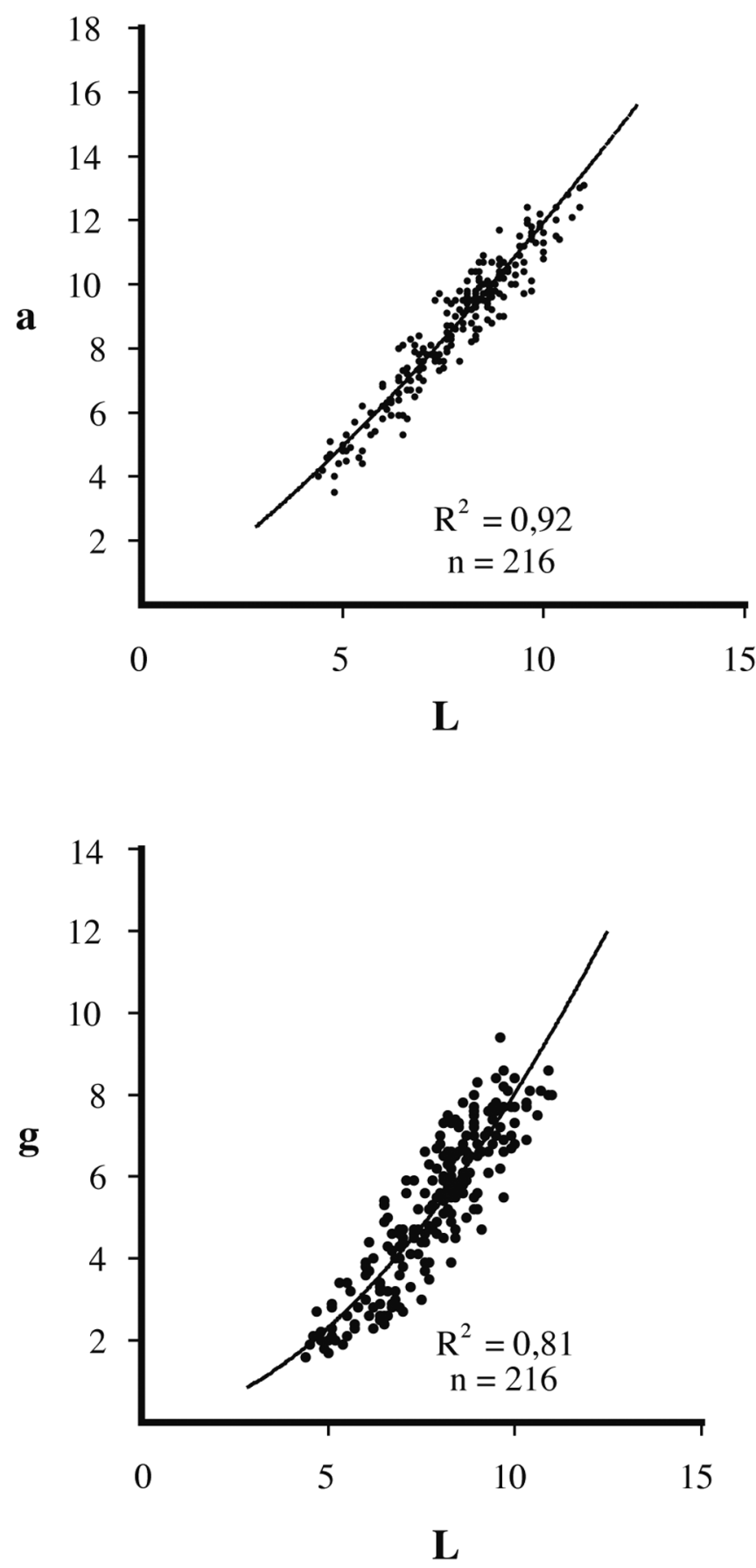

Figura 12. Diagramas de dispersión longitud/anchura (L/a) y longitud/grosor (L/g) de Iberirhynchia santaluciensis Drot \& Westbroek, 1966.

Iberirhynchia santaluciensis Drot \& Westbroek, 1966. Length/Width (L/a), and Length/Thickness $(L / g)$ dispersion diagrams. algún elemento radial, disminución de la anchura de las costillas y otras anomalías (Figs. 1.d1-d4, 2.c1-c2, 13).

Placas dentales relativamente largas (las bases se prolongan más allá de la plataforma cardinal, incluso hasta la mitad de la longitud), robustas y verticales, delimitando cavidades apicales laterales estrechas y libres y una gran cavidad apical central. Los bordes cardinales se extienden en sendas proyecciones agudas flanqueando los dientes cardinales. Dientes cardinales masivos, con la superficie denticulada por elementos secundarios y bases excavadas para alojar los extremos distales de las crestas internas de las cavidades glenoideas (Figs. 4-6); éstas, son muy altas y desarrolladas en dirección ventral (Figs. 3-6). El fondo de la valva dorsal tiende a elevarse por aposición de finas láminas de concha secundaria, formando un seudosepto medio (Fig. 5.a-c) robusto que alcanza alrededor de la mitad de la longitud; este desarrollo se refleja exteriormente en la existencia de la depresión media umbonal descrita anteriormente, la cual suele prolongarse hacia delante, en muchos ejemplares, hasta el mismo borde anterior de la valva. Las placas crurales se unen entre sí y a los márgenes distales del tabique formando un seudoseptalio bastante amplio, cubierto hacia delante por un conectivo bien desarrollado, de techo agudo, que se prolonga, más allá del propio seudoseptalio, entre las bases crurales (Fig. 3-6). Las cavidades apicales laterales dorsales son amplias y libres. Los crura, de sección triangular, se extienden en el plano comisural, relativamente juntos, durante una parte de su recorrido, para transformarse luego en laminillas aplanadas que divergen hacia delante en dirección ventral (Figs. 4.a, 6).

Observaciones paleoecológicas (basadas esencialmente en la colección propia de material, probablemente topotípico, del corte de la pista a lo largo del arroyo de La Trapa, ver más arriba).

El crecimiento de las conchas es alométrico, con un aumento proporcionalmente mayor de anchura y grosor, respecto a longitud, con la edad de los ejemplares (Figs. 12-13).

La forma de las conchas cambia de manera considerable en el curso del desarrollo. Las fases protegular y bréfica son inapreciables en el material disponible. En cambio, se dispone de individuos neánicos, efébicos y gerónticos, aunque la proporción relativa no es indicativa de mortalidad normal y, con toda probabilidad, muchos cadáveres de individuos juveniles debieron de ser alejados de la asociación antes del enterramiento, o destruidos en el curso de la fosilización. Los individuos neánicos comprenden conchas con longitudes entre 4,5 y $6 \mathrm{~mm}$ aunque, excepcionalmente, individuos de mayor talla conservan caracteres juveniles (conchas hasta de 7,4 mm de longitud), alargadas a equidimensionales ( $\mathrm{a} / \mathrm{L}$ entre 0,90 y 1 ), ventribiconvexas y muy delgadas, con comisuras cortantes, gancho débilmente inclinado y ángulo apical agudo (40 a 60 , rara vez más), seno ventral y pliegue medio dorsal no desarrollados (Figs 1.a1-a4, 13).

El paso al estadio efébico se marca por la existencia de una fuerte lamela de crecimiento, que indica una detención más o menos importante en el desarrollo. Esta discontinuidad, presente en más del $65 \%$ de los ejempla- 

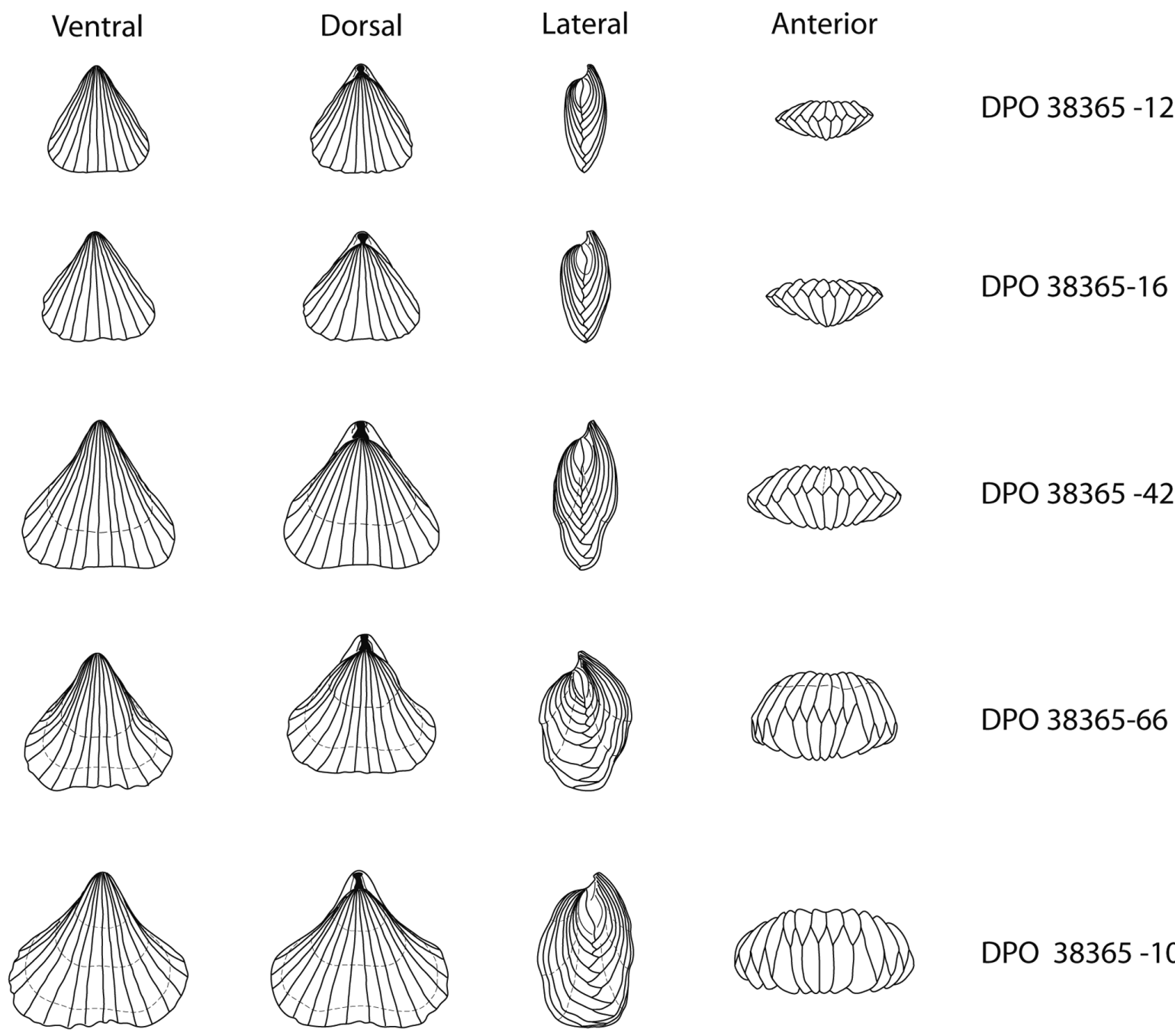

DPO $38365-104$
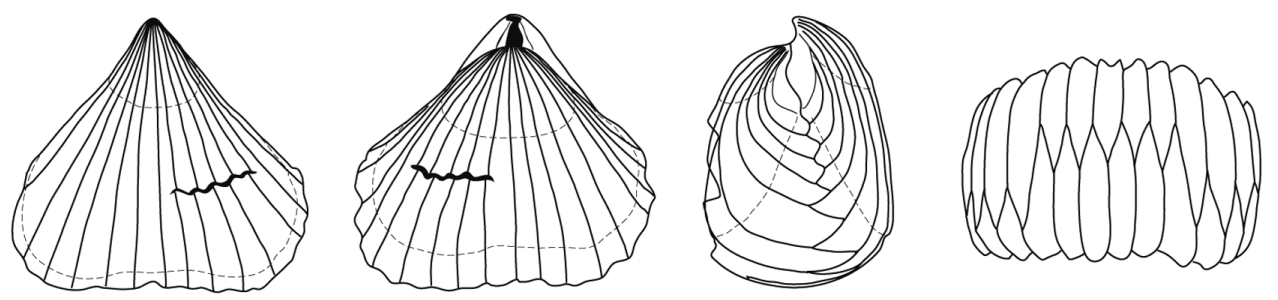

DPO 38365-205
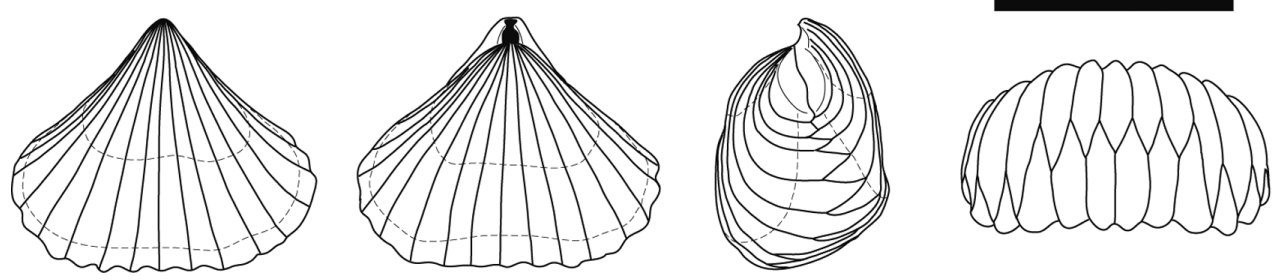

DPO 38365-208

Figura 13. Dibujos esquemáticos con cámara clara de una serie ontogenética de Iberirhynchia santaluciensis Drot \& Westbroek, 1966. Obsérvense las relaciones alométricas L/a y L/g (ver también Fig. 11). El ejemplar 205 muestra una importante reparación de la concha a partir de la cual el desarrollo ulterior de los elementos radiales presenta un notorio desfase (ver también Fig. 1.d1).

Camera lucida schematic drawings of an Iberirhynchia santaluciensis Drot \& Westbroek, 1966, ontogenetic series. See L/a and L/g allometric relations (see also Fig. 11). The specimen 205 shows an important shell repair, and in front of it a marked displacement of the radial rib growing vectors (see also Fig. 1.d1). 
res, tiende a borrarse en las conchas efébicas tardías pero es muy evidente en las efébicas tempranas (Figs. 1.d1-d4, 13). La fase madura (efébica) se caracteriza por la presencia de conchas cada vez más transversas, gruesas, dorsibiconvexas, con ganchos suberectos y ángulos apicales cada vez mayores, seno ventral y pliegue medio dorsal bien desarrollados y comisuras obtusas, con paredes laterales verticalizadas (Figs. 1.e1-e4, 13). Este estadio comienza en diferentes momentos del crecimiento en los distintos individuos, pero la mayor frecuencia se registra hacia los 5,5-6 $\mathrm{mm}$ de longitud.

El estadio geróntico (efébico tardío), suele estar representado por conchas relativamente muy largas (más de 9 $\mathrm{mm}$ de longitud, hasta cerca de $11 \mathrm{~mm}$ ), muy altas y transversas $(\mathrm{a} / \mathrm{L}$ con valores superiores a 1,20 hasta 1,35 como máximo), con flancos laterales altos y abruptos que se encuentran en la comisura en ángulo de $180^{\circ}$, ángulo apical casi recto (con valores entre 70 y $90^{\circ}$, hasta $93^{\circ}$ como máximo). Esta fase suele iniciarse en una nueva detención del crecimiento (hacia 7 a $9 \mathrm{~mm}$ de longitud), marcada por una lamela más fuerte que las otras, a partir de la cual, la curvatura de la concha suele cambiar apreciablemente haciéndose abrupta hacia las comisuras (Figs. 2, 13).

El número de costillas radiales crece poco con la edad y no existe variación significativa en el número de costillas de la parte media de la concha en la fase efébica, aunque sí la hay, lógicamente, en la relación entre la anchura del seno ventral y la de la concha que aumenta con la edad (desde valores en torno al 60\% en las conchas efébicas tempranas a otros en torno al $70 \%$, en las tardías). La mayoría de los individuos (más del 80\%) tienen una fórmula costal media de $6 / 5$ y pequeños porcentajes de ejemplares, presentan fórmulas 7/6 (10\%), 8/7 y 5/4 (alrededor del 3\%) y $9 / 8$ (un solo individuo de 217 analizados). No se observaron costillas parietales. El número de costillas laterales oscila de 12 a 20 , aunque este conteo tiene un margen de error debido al debilitamiento de los elementos laterales.

Alrededor del 15\% de las conchas estudiadas presentaba epizoos, principalmente tubos de serpúlidos, y pequeñas colonias de corales tabulados y briozoos. La colonización debió ser en muchos casos de tipo comensalista sin cambios aparentes en el huésped, pero a veces aparecen alteraciones patológicas en los vectores de crecimiento, que indican una simbiosis in vivo que, en todo caso, no parece haber sido casi nunca letal para el huésped a corto término. La presencia relativamente pequeña de epizoos, puede ser indicativa de un fondo bastante estable, donde el peligro de hundimiento de los pequeños organismos bentónicos no era muy grande.

Unos pocos individuos (Fig. 1.d1 y 13, DPO 38365205 , por ejemplo) muestran señales de roturas reparadas. El pequeño porcentaje de reparaciones y de epizoos y el abrumadoramente alto de conchas completas, en bastante buen estado (salvo alteraciones imputables a la diagénesis), acreditan medios de escasa energía, probablemente protegidos por edificios biohermales coralinos de pequeño tamaño. Las reparaciones conllevan, con frecuencia, anomalías en el crecimiento posterior, como, por ejemplo, el desfase en la dirección de crecimiento de las costillas radiales (Fig. 1.d1, 13), la supresión de una costilla o la presencia de una cicatriz extensa carente de elementos radiales. La frontera entre las fases neánica y madura es, a veces, una clara flexión de los márgenes de la concha que presagia el posterior desarrollo en altura de la concha efébica (Fig. 13); esta curvatura puede acentuarse tanto que debilite el área circundante, lo que hace que varias de las roturas observadas se encuentren en el límite entre las fases de crecimiento (Fig.1.d1). Las costillas se desarrollan en anchura y altura hacia delante, pero cuando se produce una detención en el crecimiento, el desarrollo ulterior es regresivo: los nuevos sectores de las costillas comienzan siendo más débiles que los precedentes, como si dichos elementos comenzasen su crecimiento ex novo, aunque la tasa de crecimiento es más rápida que antes y pronto se alcanza y se supera el desarrollo de las costillas en el frente de la lamela engrosada precedente (Fig. 1.c2, 2.a1).

Discusión: I. santaluciensis es una especie muy característica, difícil de confundir con ninguna otra, como ya advirtieron sus autores. De I. nargis Havlíček \& Kukal, 1990 e Iberirhynchia sp. Havlíček, 1992 se diferencia claramente en la posesión de seudoseptalio completo, cubierto por un conectivo. Ambas formas bohémicas, con septo medio dorsal fino, provisto de mediotest y carentes de conectivo, pertenecen a la Familia Trigonirhynchiidae, la primera de ellas posiblemente al propio género Nymphorhynchia donde originalmente la incluyó Havlíček (1961).

Edad: Los diferentes autores que mencionaron la especie, incluido el presente autor (Drot \& Westbroek, 1966, García-Alcalde 1990 -in Truyols et al.-, 1995, 1996, 2001 y 2006 -in Ellwood et al.-), le asignaron una edad Emsiense, frecuentemente sin más precisión. Los nuevos muestreos realizados indican que la especie se acantona en un espacio estratigráfico reducido, cercano al límite operativo Emsiense-Eifeliense, en el dominio Astur-Leonés de la Cordillera Cantábrica. Dicho límite se encuentra inmediatamente debajo de la primera aparición del conodonto Icriodus retrodepressus, probablemente en la parte alta de la Zona de Polygnatus costatus patulus, tal como pudo contrastarse indirectamente con datos bioestratigráficos y magnetoestratigráficos conjugados (Ellwood et al., 2006). Esta distribución parece general a lo largo del ámbito de aparición de la especie, lo que la convierte en una excelente guía estratigráfica para detectar la proximidad de la transición Emsiense-Eifeliense (Devónico Inferior/Devónico Medio) en el dominio facial nerítico cantábrico, donde no aparecen los indicadores estratigráficos formales de la base del Devónico Medio. 


\section{Subfamilia Myrmirhynxinae nov. subfam.}

Género tipo: Myrmirhynx Havlíček, 1982.

Diagnosis: Shell small; subtriangular to subpentagonal outline. Costae strong, increasing by bifurcation and intercalation; ventral sulcus and dorsal fold low but distinct; umbonal depression strongly marked turning anteriorly into a strong median sulcus of the dorsal fold; anterior commissure sulciplicate. Dental plates slightly converging ventrally toward valve floor. Pseudoseptalium short, with cover plate.

Iberirhynchiidae de pequeño tamaño; contorno subtriangular a subpentagonal. Costillas fuertes, creciendo hacia delante por bifurcación e intercalación Pliegue dorsal y seno ventral poco desarrollados pero bien definidos. Depresión umbonal marcada, que se transforma hacia delante en un fuerte surco medio del pliegue dorsal; comisura anterior surcoplegada. Placas dentales convergiendo algo ventralmente. Seudoseptalio corto, cubierto.

Géneros asignados: Además del tipo, se incluye en esta subfamilia el género Sufetirhynchia Havlíček, 1982.

Edad y filogenia: Los Myrmirhynxinae constituyen un pequeño stock de formas del Silúrico de Bohemia (República Checa), especialmente del Ludloviense (Silúrico superior) de la Formación Kopanina, de afinidades desconocidas, que habría derivado, quizás, de los Virginiatinae Amsden, 1974 por desarrollo de seudoseptalio cubierto. Los Myrmirhynxinae muestran características que tienden a aparecer en distintos linajes ancistrorincoideos, sobre todo en los más antiguos, por ejemplo la ornamentación radial dividida, con pliegues robustos y líneas de crecimiento marcadas. Los Niorhynicidae Savage, 1996 presentan también ornamentación radial con pliegues fuertes y divididos, y seno ventral y pliegue dorsal bajos, como en Myrmirhynxinae, pero carecen de placas dentales y el seudoseptalio no siempre está cubierto

\section{AGRADECIMIENTOS}

Mi profundo agradecimiento al doctor Cornelius F. Winkler Prins y al Rijksmuseum van Geologie en Mineralogie, de Leiden, Holanda, por el préstamo de parte del material original de la especie Iberirhynchia santaluciensis. Al propio Dr. Winkler Prins y a los doctores Peter Floor, Peter Westbroek y José R. Vidal Romaní, que realizaron diversas gestiones, por desgracia infructuosas, para localizar en Holanda y España las tesinas de los estudiantes de Leiden que recogieron el material original de la especie.

Mi gratitud también para las revisoras, Dras. Denise Brice, de la Universidad de Lille, Francia y María L. Martínez-Chacón, de la de Oviedo, por su minuciosa corrección y excelentes sugerencias, que mejoraron notablemente el texto original. La Dra.
Denise Brice me facilitó, además, una réplica de material topotípico de Trigonirhynchia fallaciosa, para ilustrar convenientemente la estructura septal propia de la Familia Trigonirhynchiidae.

El trabajo forma parte del proyecto PICG 499 "Evolución de los ecosistemas y climas durante el Devónico", y fue financiado por el proyecto MEC-05-CGL 2005-03715 "Fases de desarrollo de las asociaciones de corales y estromatoporoideos en el Devónico Inferior de la Cordillera Cantábrica (NO de España)" y Fondos FEDER.

\section{BIBLIOGRAFÍA}

Alonso, J.L. 1985. Estructura y evolución tectonoestratigráfica de la región del manto del Esla (Zona Cantábrica, NW de España). Institución Fray Bernardino de Sahagún (Diputación Provincial de León), 276 pp.

Amsden, T.W. 1974. Late Ordovician and Early Silurian articulate brachiopods from Oklahoma, southwestern Illinois, and eastern Missouri. Oklahoma Geological Survey Bulletin, 119, 1-154.

Cooper, G.A. 1942. New genera of North American brachiopods. Journal of the Washington Academy of Sciences, 32, 228-235.

Cooper, G.A. 1956. Chazian and related brachiopods. Smithsonian Miscellaneous Collections, 127, 1-1245.

Drot, J. \& Westbroek, P. 1966. Iberirhynchia santaluciensis, nouveau rhynchonellacea du Dévonien de Léon (Espagne). Leidse Geologische Mededelingen, 38, 165-172.

Ellwood, B.B., García-Alcalde, J.L., El Hassani, A., Hladil, J., Soto, F.M., Truyols-Massoni, M., Weddige, K. \& Koptikova, L. 2006. Stratigraphy of the Middle Devonian boundary: formal definition of the susceptibility magnetostratotype in Germany with comparisons to sections in the Czech Republic, Morocco and Spain. Tectonophysics, 418, 31-49.

García-Alcalde, J.L. 1995. L'évolution paléogéographique pre-varisque de la zone Cantabrique septentrionale (Espagne). Revista Española de Paleontología, 10, 9-29.

García-Alcalde, J.L. 1996. El Devónico del dominio AsturLeonés en la Zona Cantábrica (N de España). Revista Española de Paleontología, $\mathbf{n}^{\mathbf{0}}$ extraordinario, 58-71.

García-Alcalde, J.L. 1998. Braquiópodos rinconélidos del Lochkoviense (Devónico Inferior) de la Cordillera Cantábrica (Norte de España). Géobios, 31, 767-789.

García-Alcalde, J.L. 2001. Paleobiogeographical relationships between North Gondwana and South Baltica: The Ivanothyris havliceki fauna (Cantabrian Zone, latest Emsian). Journal of the Czech Geological Society, 46, 121-130.

García-Alcalde, J.L. 2004 Lower Devonian Delthyridoidea (Brachiopoda, Delthyridina) of the Cantabrian Mountains (N Spain). Bulletin de l'Institut royal des Sciences naturelles de Belgique, 74, supplement, 9-38.

García-Alcalde, J.L. 2005. Paleobiogeografía de Cyrtinopsidae (Braquiópodos Delthyridina) del Silúrico y Devónico. Revista Española de Paleontología, 20, 169-176.

Havlíček, V. 1959. Rhynchonellacea im böhmischen älteren Paläozoikum (Brachiopoda). Vestník Ústředního ústavu geologického, 34, 78-82. 
Havlíček, V. 1961. Rhynchonelloidea des böhmischen älteren Paläozoikums (Brachiopoda). Rozpravy Ústřední ústavu geologického, 27, 1-211.

Havlíček, V. 1982. New genera of rhynchonellid and camerellid brachiopods in the Silurian of Bohemia. Vestník Ústředního ústavu geologického, 57, 365-372.

Havliček, V. 1992. New Lower Devonian (Lochkovian-Zlichovian) rhynchonellid brachiopods in the Prague Basin. Sborník geologických ved. Paleontologie, 32, 55-122.

Havlíček, V. \& Kukal, Z. 1990. Sedimentology, benthic communities, and brachiopods in the Suchomasty (Dalejan) and Acanthopyge (Eifelian) Limestones osf the Koneprusy area (Czechoslovakia). Sbornik geologických ved. Paleontologie, 31, 105-205.

Havlíček, V. \& Štorch, P. 1990. Silurian brachiopods and benthic communities in the Prague Basin (Czechoslovakia). Rozpravy Ústředního, ústavu geologického, 48, 1-275.

Kuhn, O. 1949. Lehrbuch der Paläozoologie. E. Schweizerbart'sche Verlagbuchhandlung, 329 pp.

Rozman, Kh.S. 1969. Pozdneordovikskie Brakhiopody Sibirskoi Platformy. Paleontologicheskii Zhurnal, 1969, 86-108.

Sartenaer, P. 1970. Nouveaux genres rhynchonellides (brachiopodes) du Paléozoique. Bulletin de l'Institut royal des Sciences naturelles de Belgique, 46, 1-32.

Savage, N.M. 1996. Classification of Paleozoic rhynchonellid brachiopods. In: Brachiopods (ed. P. Copper \& J. Jin). Proceedings 3 International Brachiopod Congress, 249-260.
Savage, N.M. 2002. Rhynchotrematoidea. In: Treatise on Invertebrate Paleontology (Ed. R.L. Kaesler). H 4, Rhynchonelliformea (part) revised, 1047-1091.

Schmidt, H. 1965. Neue befunde an Paläozoischen Rhynchonellacea (Brachiopoda). Senckenbergiana Lethaea, 46, $1-25$.

Schuchert, Ch. 1913. Class 2. Brachiopoda. In: Text-book of Palaeontology (K.A. von Zittel) (ed. C.R. Eastman), 1, 355-420.

Schumann, D. 1965. Rhynchonelloidea aus dem Devon des Kantabrischen Gebirges (Nordspanien). Neues Jahrbuch Geologie und Paläontologie Abhandlungen, 123, 41-104.

Truyols, J., Arbizu, M.A., García-Alcalde, J.L., García-López, S., Méndez-Bedia, I., Soto, F. \& Truyols-Massoni, M. 1990. The Asturian-Leonese Domain (Cantabrian Zone) In: Pre-Mesozoic geology of Iberia (Ed. R.D. Dallmeyer \& E. Martínez-García), Springer-Verlag, 10-19.

Ulrich, E.O. \& Cooper, G.A. 1942. New genera of Ordovician brachiopods. Journal of Paleontology, 16, 620-626.

Westbroek, P. 1967. Morphological observations with systematic implications on some Palaeozoic Rhynchonellida from Europe, with special emphasis on the Uncinulidae. Leidse Geologische Mededelingen, 41, 1-82.

Manuscrito recibido: 24 de Septiembre, 2009 Manuscrito aceptado: 22 de Diciembre, 2009 


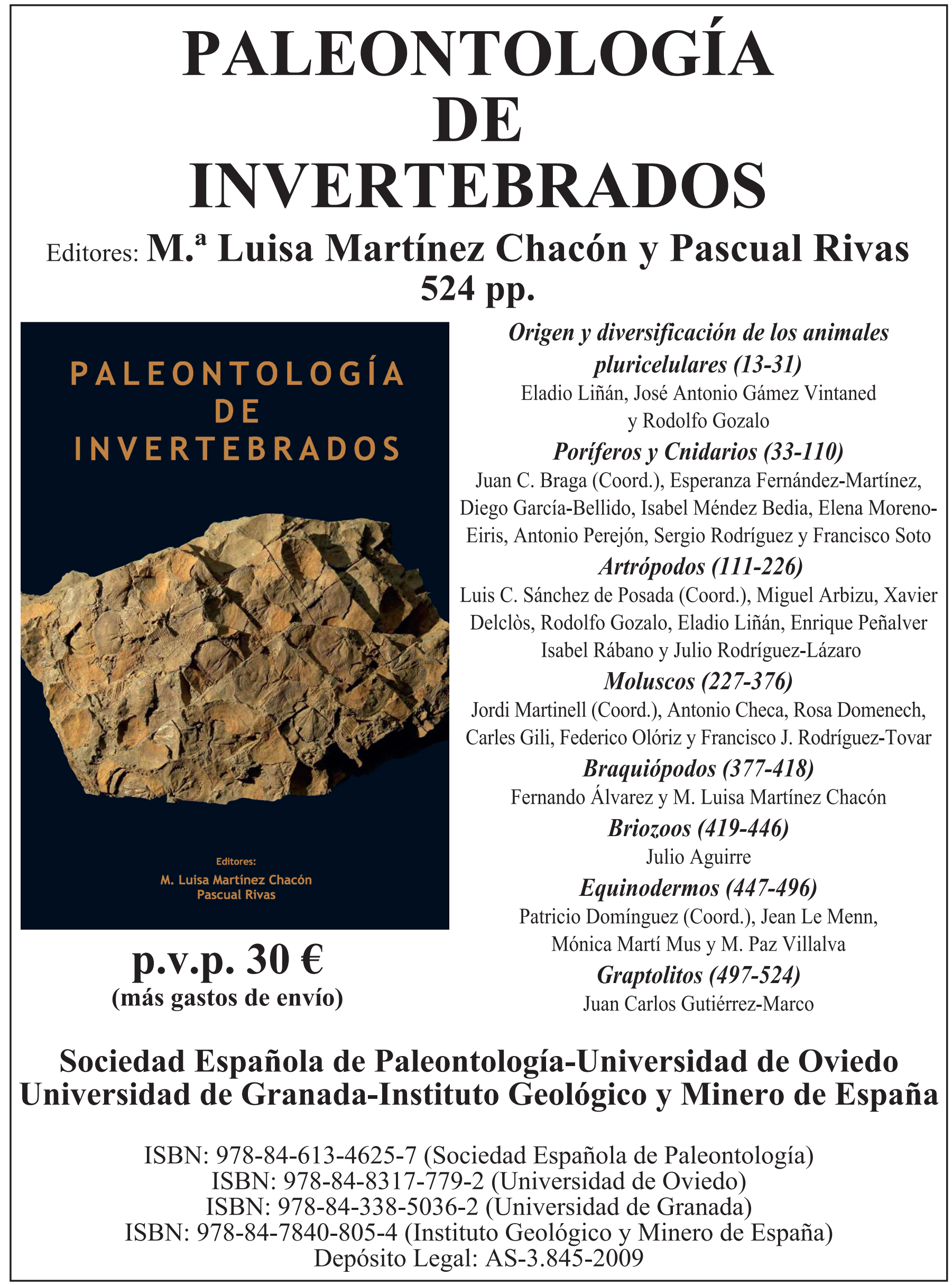

